\title{
Analytical solutions for the static analysis of laminated composite and sandwich plates based on a higher order refined theory
}

\author{
T. Kant*, K. Swaminathan \\ Department of Civil Engineering, Indian Institute of Technology Bombay, Powai, Mumbai 400 076, India
}

\begin{abstract}
Analytical formulations and solutions to the static analysis of simply supported composite and sandwich plates hitherto not reported in the literature based on a higher order refined theory developed by the first author and already reported in the literature are presented. The theoretical model presented herein incorporates laminate deformations which account for the effects of transverse shear deformation, transverse normal strain/stress and a nonlinear variation of in-plane displacements with respect to the thickness coordinate - thus modelling the warping of transverse cross-sections more accurately and eliminating the need for shear correction coefficients. In addition, a few higher order theories and the first order theory developed by other investigators and already available in the literature are also considered for the evaluation. The equations of equilibrium are obtained using principle of minimum potential energy (PMPE). Solutions are obtained in closed form using Navier's technique by solving the boundary value problem. The comparison of the present results with the available elasticity solutions and the results computed independently using the first order and the other higher order theories available in the literature shows that this refined theory predicts the transverse displacement and the stresses more accurately than all other theories considered in this paper. After establishing the accuracy of present results for composite and sandwich plates, new results for the stretching-bending coupling behaviour of antisymmetric sandwich laminates using all the theories considered in this paper are presented which will serve as a benchmark for future investigations.
\end{abstract}

Keywords: Static analysis; Higher-order theory; Shear deformation; Sandwich plates; Analytical solutions

\section{Introduction}

Laminated composite plates are being increasingly used in the aeronautical and aerospace industry as well as in other fields of modern technology. To use them efficiently a good understanding of their structural and dynamical behaviour and also an accurate knowledge of the deformation characteristics, stress distribution, natural frequencies and buckling loads under various load conditions are needed. The classical laminate plate theory (CLPT) [1], which is an extension of classical plate theory (CPT) [2,3], neglects the effects of outof-plane strains. The greater differences in elastic properties between fibre filaments and matrix materials lead to a high ratio of in-plane young's modulus to transverse shear modulus for most of the composite laminates developed to date. Because of this reason the transverse shear deformations are much pronounced for laminated plates than for isotropic plates. Thus the CLPT which ignores the effect of transverse shear deformation becomes inadequate for the analysis of multilayer composites. In general the CLPT often underpredicts deflections and overpredicts natural frequencies and buckling loads. The first order theories (FSDTs) based on Reissner [4] and Mindlin [5] assume linear in-plane stresses and displacements, respectively through the laminate thickness. Since the FSDT accounts for layerwise constant states of transverse shear stress, shear correction coefficients are needed to rectify the unrealistic variation of the shear strain/stress through the thickness and which ultimately define the shear strain energy.

In order to overcome the limitations of FSDT, higher order shear deformation theories (HSDTs) that involve higher order terms in Taylor's expansions of the displacements in the thickness coordinate were developed. In these higher order theories with each additional power of the thickness coordinate an 
additional dependent unknown is introduced into the theory. Hildebrand et al. [6] were the first to introduce this approach to derive improved theories of plates and shells. Nelson and Lorch [7], Librescu [8] presented higher order displacement based shear deformation theories for the analysis of laminated plates. Lo et al. $[9,10]$ have presented a closed form solution for a laminated plate with higher order displacement model which also considers the effect of transverse normal deformation. Levinson [11] and Murthy [12] presented third order theories neglecting the extension/ compression of transverse normal but used the equilibrium equations of the first order theory used by Whitney and Pagano [13] in the analysis which are variationally inconsistent. Kant [14] was the first to derive the complete set of variationally consistent governing equations for the flexure of a symmetrically laminated plate incorporating both distortion of transverse normals and effects of transverse normal stress/strain by utilizing the complete three-dimensional generalized Hooke's law and presented results for isotropic plate only. Reddy [15] derived a set of variationally consistent equilibrium equations for the kinematic models originally proposed by Levinson and Murthy. Using the theory of Reddy, Senthilnathan et al. [16] presented a simplified higher order theory by introducing a further reduction of the functional degrees of freedom by splitting up the transverse displacement into bending and shear contributions. Kant et al. [17] are the first to present a finite element formulation of a higher order flexure theory. This theory considers three-dimensional Hooke's law, incorporates the effect of transverse normal strain in addition to transverse shear deformations. Pandya and Kant [18-22], Kant and Manjunatha [23,24] and Manjunatha and Kant [25] have extended this theory and presented $C^{0}$ finite element formulations and solutions for the stress analysis of symmetric and unsymmetric laminated composite and sandwich plates. Rohwer [26] made a comparative study of various higher order theories for the bending analysis of multilayer composite plates. The advantages and disadvantages of the various theories were highlighted. Noor and Burton [27] presented a complete list of references of FSDTs and HSDTs for the static, free vibration and buckling analyses of laminated composites. Pagano [28] presented exact three dimensional elasticity solutions for the stress analysis of laminated composite and sandwich plates which serve as benchmark solutions for comparison by many researchers. The present paper deals with the analytical formulations and solutions hitherto not reported in the literature of the refined theory already proposed by the senior author as applied to static analysis of laminated composite and sandwich plate problems with simply supported edge conditions. Comparison of results with the three-dimensional elasticity solutions available in the literature shows that this theory predicts the transverse displacements and the in plane stresses more accurately than all other theories considered in this paper. After establishing the accuracy of the present results for composite and sandwich plates, benchmark results for stretching-bending coupling behaviour of antisymmetric sandwich plates are presented.

\section{Theoretical formulation}

\subsection{Displacement models}

In order to approximate the three-dimensional elasticity problem to a two-dimensional plate problem, the displacement components $u(x, y, z), v(x, y, z)$ and $w(x, y, z)$ at any point in the plate space are expanded in a Taylor's series in terms of the thickness coordinate. The elasticity solution indicates that the transverse shear stresses vary parabolically through the plate thickness. This requires the use of a displacement field in which the in-plane displacements are expanded as cubic functions of the thickness coordinate. In addition, the transverse normal strain may vary nonlinearly through the plate thickness. The displacement field which satisfies the above criteria may be assumed in the form [23]:

$$
\begin{aligned}
u(x, y, z)= & u_{0}(x, y)+z \theta_{x}(x, y) \\
& +z^{2} u_{0}^{*}(x, y)+z^{3} \theta_{x}^{*}(x, y), \\
v(x, y, z)= & v_{0}(x, y)+z \theta_{y}(x, y) \\
& +z^{2} v_{0}^{*}(x, y)+z^{3} \theta_{y}^{*}(x, y), \\
w(x, y, z)= & w_{0}(x, y)+z \theta_{z}(x, y) \\
& +z^{2} w_{0}^{*}(x, y)+z^{3} \theta_{z}^{*}(x, y) .
\end{aligned}
$$

Further if the variation of transverse displacement 2component $w(x, y, z)$ in Eq. (1) is assumed constant through the plate thickness and thus setting $\varepsilon_{z}=0$, then the displacement field may be expressed as [22]

$$
\begin{aligned}
u(x, y, z)= & u_{0}(x, y)+z \theta_{x}(x, y) \\
& +z^{2} u_{0}^{*}(x, y)+z^{3} \theta_{x}^{*}(x, y), \\
v(x, y, z)= & v_{0}(x, y)+z \theta_{y}(x, y) \\
& +z^{2} v_{0}^{*}(x, y)+z^{3} \theta_{y}^{*}(x, y), \\
w(x, y, z)= & w_{0}(x, y) .
\end{aligned}
$$

The parameters $u_{0}, v_{0}$ are the in-plane displacements and $w_{0}$ is the transverse displacement of a point $(x, y)$ on the middle plane. The functions $\theta_{x}, \theta_{y}$ are rotations of the normal to the middle plane about $y$ and $x$ axes, respectively. The parameters $u_{0}^{*}, v_{0}^{*}, w_{0}^{*}, \theta_{x}^{*}, \theta_{y}^{*}, \theta_{z}^{*}$ and $\theta_{z}$ are the higher-order terms in Taylor's series expansion 
and they represent higher-order transverse cross-sectional deformation modes. Though the above two theories were already reported earlier in the literature and numerical results were presented using finite element formulations, analytical formulations and solutions are obtained for the first time in this investigation and so the results obtained using the above two theories are referred to as present (Model-1 and Model-2) in all the tables and figures. In addition to the above, the following higher order theories and the first order theory developed by other investigators and reported in the literature for the analysis of laminated composite and sandwich plates are also considered for the evaluation. Analytical formulations and numerical results of these are also being presented here with a view to have all the results on a common platform.

Model-3 [29]

$$
\begin{aligned}
u(x, y, z)= & u_{0}(x, y) \\
& +z\left[\theta_{x}(x, y)-\frac{4}{3}\left(\frac{z}{h}\right)^{2}\left\{\theta_{x}(x, y)+\frac{\partial w_{0}}{\partial x}\right\}\right], \\
v(x, y, z)= & v_{0}(x, y) \\
& +z\left[\theta_{y}(x, y)-\frac{4}{3}\left(\frac{z}{h}\right)^{2}\left\{\theta_{y}(x, y)+\frac{\partial w_{0}}{\partial y}\right\}\right], \\
w(x, y, z)= & w_{0}(x, y) .
\end{aligned}
$$

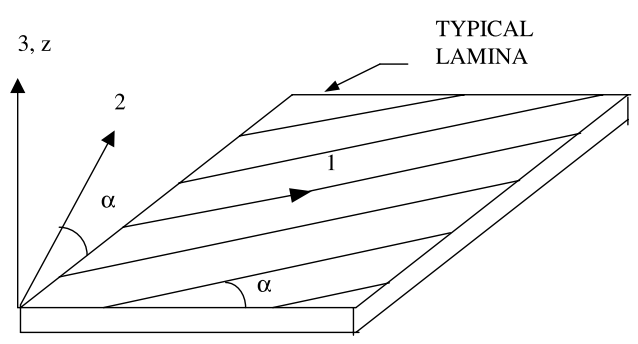

( $1,2,3)$ - LAMINA REFERENCE AXES

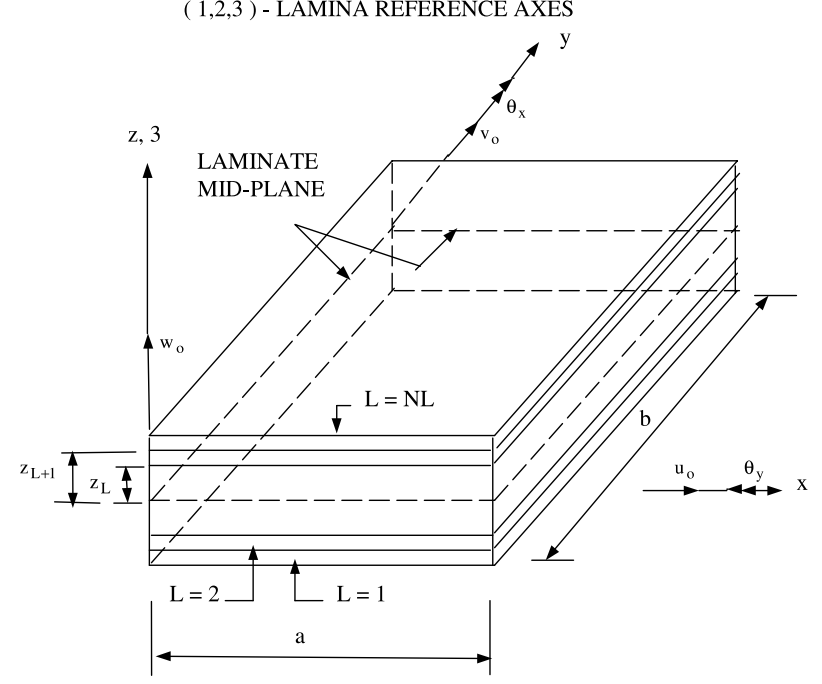

$(\mathrm{x}, \mathrm{y}, \mathrm{z})$ - LAMINATE REFERENCE AXES

Fig. 1. Laminate geometry with positive set of lamina/laminate reference axes, displacement components and fibre orientation.
Model-4 [16]

$u(x, y, z)=u_{0}(x, y)-z \frac{\partial w_{0}^{b}}{\partial x}-\frac{4 z^{3}}{3 h^{2}} \frac{\partial w_{0}^{s}}{\partial x}$,
$v(x, y, z)=v_{0}(x, y)-z \frac{\partial w_{0}^{b}}{\partial y}-\frac{4 z^{3}}{3 h^{2}} \frac{\partial w_{0}^{s}}{\partial y}$,
$w(x, y, z)=w_{0}^{b}(x, y)+w_{0}^{s}(x, y)$.

Model-5 [13]

$$
\begin{aligned}
& u(x, y, z)=u_{0}(x, y)+z \theta_{x}(x, y), \\
& v(x, y, z)=v_{0}(x, y)+z \theta_{y}(x, y), \\
& w(x, y, z)=w_{0}(x, y) .
\end{aligned}
$$

In this paper the analytical formulations and solution method followed using the higher order refined theory (Model-1) are only presented in detail and the same procedure is followed in obtaining the results using other models. The geometry of a two-dimensional laminated composite and sandwich plates with positive set of coordinate axes and the physical middle plane displacement terms are shown in Figs. 1 and 2, respectively. By substitution of the displacement relations given by Eq. (1) into the strain-displacement equations of the

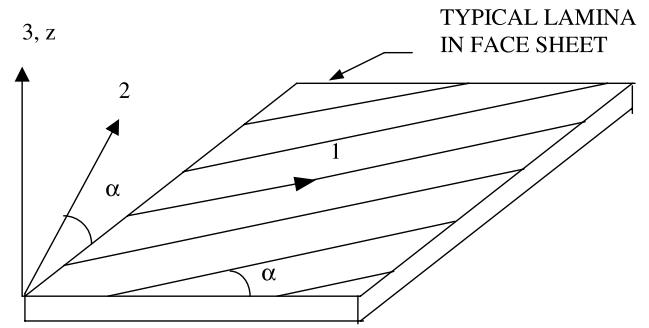

( $1,2,3$ ) - LAMINA REFERENCE AXES

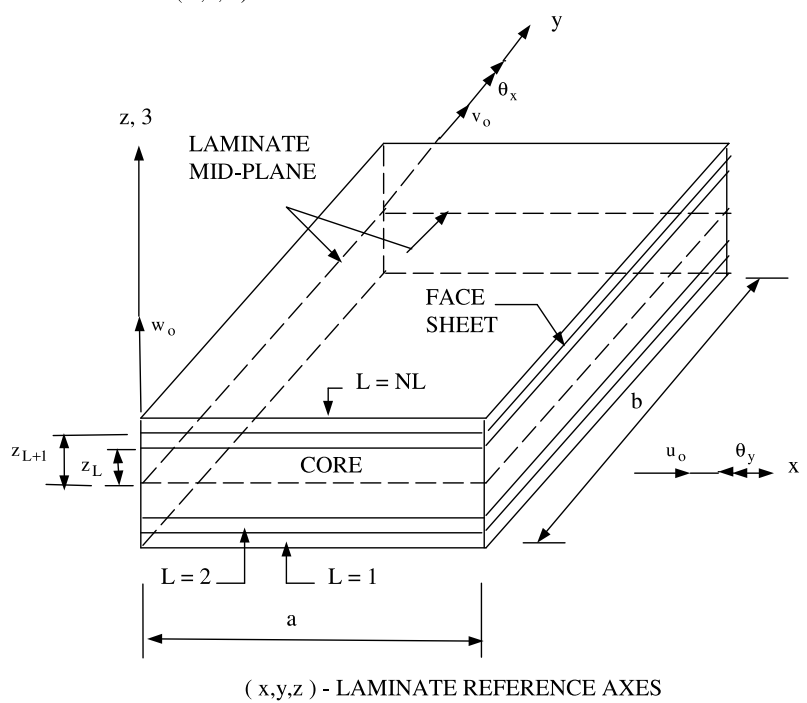

Fig. 2. Geometry of a sandwich plate with positive set of lamina/ laminate reference axes, displacement components and fibre orientation. 
classical theory of elasticity, the following relations are obtained:

$$
\begin{aligned}
& \varepsilon_{x}=\varepsilon_{x 0}+z \kappa_{x}+z^{2} \varepsilon_{x 0}^{*}+z^{3} \kappa_{x}^{*}, \\
& \varepsilon_{y}=\varepsilon_{y 0}+z \kappa_{y}+z^{2} \varepsilon_{y 0}^{*}+z^{3} \kappa_{y}^{*}, \\
& \varepsilon_{z}=\varepsilon_{z 0}+z \kappa_{z}+z^{2} \varepsilon_{z 0}^{*}+z^{3} \kappa_{y}^{*}, \\
& \gamma_{x y}=\varepsilon_{x y 0}+z \kappa_{x y}+z^{2} \varepsilon_{x y 0}^{*}+z^{3} \kappa_{x y}^{*}, \\
& \gamma_{y z}=\phi_{y}+z \kappa_{y z}+z^{2} \phi_{y}^{*}+z^{3} \kappa_{y z}^{*}, \\
& \gamma_{x z}=\phi_{x}+z \kappa_{x z}+z^{2} \phi_{x}^{*}+z^{3} \kappa_{x z}^{*},
\end{aligned}
$$

where

$$
\begin{aligned}
& \left(\varepsilon_{x 0}, \varepsilon_{y 0}, \varepsilon_{x y 0}\right)=\left(\frac{\partial u_{0}}{\partial x}, \frac{\partial v_{0}}{\partial y}, \frac{\partial u_{0}}{\partial y}+\frac{\partial v_{0}}{\partial x}\right), \\
& \left(\varepsilon_{x 0}^{*}, \varepsilon_{y 0}^{*}, \varepsilon_{x y 0}^{*}\right)=\left(\frac{\partial u_{0}^{*}}{\partial x}, \frac{\partial v_{0}^{*}}{\partial y}, \frac{\partial u_{0}^{*}}{\partial y}+\frac{\partial v_{0}^{*}}{\partial x}\right), \\
& \left(\varepsilon_{z 0}, \varepsilon_{z 0}^{*}\right)=\left(\theta_{z}, 3 \theta_{z}^{*}\right), \\
& \left(\kappa_{x}, \kappa_{y}, \kappa_{z}, \kappa_{x y}\right)=\left(\frac{\partial \theta_{x}}{\partial x}, \frac{\partial \theta_{y}}{\partial y}, 2 w_{0}^{*}, \frac{\partial \theta_{x}}{\partial y}+\frac{\partial \theta_{y}}{\partial x}\right), \\
& \left(\kappa_{x}^{*}, \kappa_{y}^{*}, \kappa_{x y}^{*}\right)=\left(\frac{\partial \theta_{x}^{*}}{\partial x}, \frac{\partial \theta_{y}^{*}}{\partial y}, \frac{\partial \theta_{x}^{*}}{\partial y}+\frac{\partial \theta_{y}^{*}}{\partial x}\right), \\
& \left(\kappa_{x z}, \kappa_{y z}\right)=\left(2 u_{0}^{*}+\frac{\partial \theta_{z}}{\partial x}, 2 v_{0}^{*}+\frac{\partial \theta_{z}}{\partial y}\right), \\
& \left(\kappa_{x z}^{*}, \kappa_{y z}^{*}\right)=\left(\frac{\partial \theta_{z}^{*}}{\partial x}, \frac{\partial \theta_{z}^{*}}{\partial y}\right), \\
& \left(\phi_{x}, \phi_{x}^{*}, \phi_{y}, \phi_{y}^{*}\right)=\left(\theta_{x}+\frac{\partial w_{0}}{\partial x}, 3 \theta_{x}^{*}+\frac{\partial w_{0}^{*}}{\partial x},\right. \\
& \left.\theta_{y}+\frac{\partial w_{0}}{\partial y}, 3 \theta_{y}^{*}+\frac{\partial w_{0}^{*}}{\partial y}\right) .
\end{aligned}
$$

\subsection{Constitutive equations}

Each lamina in the laminate is assumed to be in a three-dimensional stress state so that the constitutive relation for a typical lamina $L$ with reference to the fibre-matrix coordinate axes (1-2-3) can be written as

$$
\left\{\begin{array}{c}
\sigma_{1} \\
\sigma_{2} \\
\sigma_{3} \\
\tau_{12} \\
\tau_{23} \\
\tau_{13}
\end{array}\right\}=\left[\begin{array}{cccccc}
C_{11} & C_{12} & C_{13} & 0 & 0 & 0 \\
C_{12} & C_{22} & C_{23} & 0 & 0 & 0 \\
C_{13} & C_{23} & C_{33} & 0 & 0 & 0 \\
0 & 0 & 0 & C_{44} & 0 & 0 \\
0 & 0 & 0 & 0 & C_{55} & 0 \\
0 & 0 & 0 & 0 & 0 & C_{66}
\end{array}\right]^{L}\left\{\begin{array}{c}
\varepsilon_{1} \\
\varepsilon_{2} \\
\varepsilon_{3} \\
\gamma_{12} \\
\gamma_{23} \\
\gamma_{13}
\end{array}\right\},
$$

where $\left(\sigma_{1}, \sigma_{2}, \sigma_{3}, \tau_{12}, \tau_{23}, \tau_{13}\right)$ are the stresses and $\left(\varepsilon_{1}, \varepsilon_{2}\right.$, $\left.\varepsilon_{3}, \gamma_{12}, \gamma_{23}, \gamma_{13}\right)$ are the linear strain components referred to the lamina coordinates $(1-2-3)$ and the $C_{i j}$ 's are the elastic constants or the elements of stiffness matrix of the $L$ th lamina with reference to the fibre axes (1-2-
$3)$. In the laminate coordinates $(x, y, z)$ the stress strain relations for the $L$ th lamina can be written as

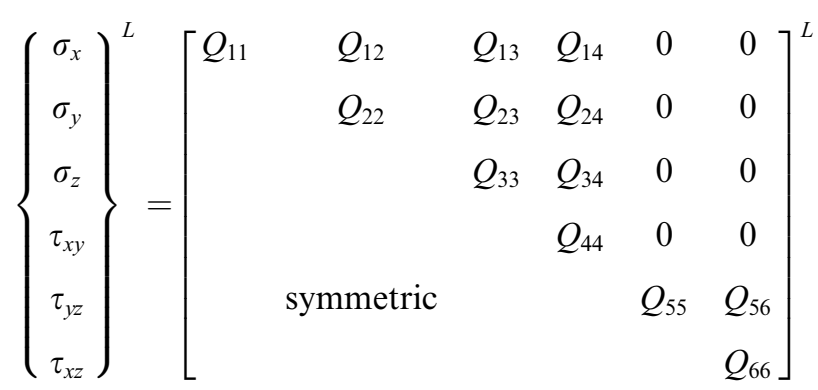

$$
\begin{aligned}
& \times\left\{\begin{array}{c}
\varepsilon_{x} \\
\varepsilon_{y} \\
\varepsilon_{z} \\
\gamma_{x y} \\
\gamma_{y z} \\
\gamma_{x z}
\end{array}\right\}
\end{aligned}
$$

where $\left(\sigma_{x}, \sigma_{y}, \sigma_{z}, \tau_{x y}, \tau_{y z}, \tau_{x z}\right)$ are the stresses and $\left(\varepsilon_{x}, \varepsilon_{y}\right.$, $\left.\varepsilon_{z}, \gamma_{x y}, \gamma_{y z}, \gamma_{x z}\right)$ are the strains with respect to the laminate axes. $Q_{i j}$ 's are the transformed elastic constants or stiffness matrix with respect to the laminate axes $x, y, z$. The elements of matrices $[C]$ and $[Q]$ are defined in Appendices A and B.

\subsection{Governing equations of equilibrium}

The equations of equilibrium for the stress analysis are obtained using the principle of minimum potential energy (PMPE). In analytical form it can be written as follows [29]:

$\delta(U+V)=0$,

where $U$ is the total strain energy due to deformations, $V$ is the potential of the external loads, and $U+V=\Pi$ is the total potential energy and $\delta$ denotes the variational symbol. Substituting the appropriate energy expression in the above equation, the final expression can thus be written as

$$
\begin{aligned}
& {\left[\int _ { - h / 2 } ^ { h / 2 } \int _ { A } \left(\sigma_{x} \delta \varepsilon_{x}+\sigma_{y} \delta \varepsilon_{y}+\sigma_{z} \delta \varepsilon_{z}+\tau_{x y} \delta \gamma_{x y}+\tau_{y z} \delta \gamma_{y z}\right.\right.} \\
& \left.\left.\quad+\tau_{x z} \delta \gamma_{x z}\right) \mathrm{~d} A \mathrm{~d} z-\int_{A} p_{z}^{+} \delta w^{+} \mathrm{d} A\right]=0,
\end{aligned}
$$

where $w^{+}=w_{0}+(h / 2) \theta_{z}+\left(h^{2} / 4\right) w_{0}^{*}+\left(h^{3} / 8\right) \theta_{z}^{*}$ is the transverse displacement of any point on the top surface of the plate and $p_{z}^{+}$is the transverse load applied at the top surface of the plate. Using Eqs. (1), (6) and (7) in Eq. (11) and integrating the resulting expression by parts, and collecting the coefficients of $\delta u_{0}, \delta v_{0}, \delta w_{0}, \delta \theta_{x}, \delta \theta_{y}$, $\delta \theta_{z}, \delta u_{0}^{*}, \delta v_{0}^{*}, \delta w_{0}^{*}, \delta \theta_{x}^{*}, \delta \theta_{y}^{*}, \delta \theta_{z}^{*}$ the following equations of equilibrium are obtained: 


$$
\begin{aligned}
\delta u_{0}: & \frac{\partial N_{x}}{\partial x}+\frac{\partial N_{x y}}{\partial y}=0 \\
\delta v_{0}: & \frac{\partial N_{y}}{\partial y}+\frac{\partial N_{x y}}{\partial x}=0 \\
\delta w_{0}: & \frac{\partial Q_{x}}{\partial x}+\frac{\partial Q_{y}}{\partial y}+p_{z}^{+}=0 \\
\delta \theta_{x}: & \frac{\partial M_{x}}{\partial x}+\frac{\partial M_{x y}}{\partial y}-Q_{x}=0 \\
\delta \theta_{y}: & \frac{\partial M_{y}}{\partial y}+\frac{\partial M_{x y}}{\partial x}-Q_{y}=0 \\
\delta \theta_{z}: & \frac{\partial S_{x}}{\partial x}+\frac{\partial S_{y}}{\partial y}-N_{z}+\frac{h}{2}\left(p_{z}^{+}\right)=0 \\
\delta u_{0}^{*}: & \frac{\partial N_{x}^{*}}{\partial x}+\frac{\partial N_{x y}^{*}}{\partial y}-2 S_{x}=0 \\
\delta v_{0}^{*}: & \frac{\partial N_{y}^{*}}{\partial y}+\frac{\partial N_{x y}^{*}}{\partial x}-2 S_{y}=0 \\
\delta \theta_{z}^{*}: & \frac{\partial S_{x}^{*}}{\partial x}+\frac{\partial S_{y}^{*}}{\partial y}-3 N_{z}^{*}+\frac{h^{3}}{8}\left(p_{z}^{+}\right)=0 \\
\delta w_{0}^{*}: & \frac{\partial Q_{x}^{*}}{\partial x}+\frac{\partial Q_{y}^{*}}{\partial y}-2 M_{z}^{*}+\frac{h^{2}}{4}\left(p_{z}^{+}\right)=0 \\
\delta \theta_{x}^{*}: & \frac{\partial M_{x}^{*}}{\partial x}+\frac{\partial M_{x y}^{*}}{\partial y}-3 Q_{x}^{*}=0 \\
\delta M_{y}^{*} & \frac{\partial M_{x y}^{*}}{\partial x}-3 Q_{y}^{*}=0 \\
\delta & =0 \\
\delta &
\end{aligned}
$$

and the boundary conditions are of the form:

On the edge $x=$ constant

$u_{0}=\bar{u}_{0}$ or $N_{x}=\bar{N}_{x}, \quad \theta_{x}=\bar{\theta}_{x}$ or $M_{x}=\bar{M}_{x}$,

$v_{0}=\bar{v}_{0}$ or $N_{x y}=\bar{N}_{x y}, \quad \theta_{y}=\bar{\theta}_{y}$ or $M_{x y}=\bar{M}_{x y}$,

$w_{0}=\bar{w}_{0}$ or $Q_{x}=\bar{Q}_{x}, \quad \theta_{z}=\bar{\theta}_{z}$ or $S_{x}=\bar{S}_{x}$,

$u_{0}^{*}=\bar{u}_{0}^{*}$ or $N_{x}^{*}=\bar{N}_{x}^{*}, \quad \theta_{x}^{*}=\bar{\theta}_{x}^{*}$ or $M_{x}^{*}=\bar{M}_{x}^{*}$,

$v_{0}^{*}=\bar{v}_{0}^{*}$ or $N_{x y}^{*}=\bar{N}_{x y}^{*}, \quad \theta_{y}^{*}=\bar{\theta}_{y}^{*}$ or $M_{x y}^{*}=\bar{M}_{x y}^{*}$,

$w_{0}^{*}=\bar{w}_{0}^{*}$ or $Q_{x}^{*}=\bar{Q}_{x}^{*}, \quad \theta_{z}^{*}=\bar{\theta}_{z}^{*}$ or $S_{x}^{*}=\bar{S}_{x}^{*}$.

On the edge $y=$ constant

$u_{0}=\bar{u}_{0}$ or $N_{x y}=\bar{N}_{x y}, \quad \theta_{x}=\bar{\theta}_{x}$ or $M_{x y}=\bar{M}_{x y}$,

$v_{0}=\bar{v}_{0}$ or $N_{y}=\bar{N}_{y}, \quad \theta_{y}=\bar{\theta}_{y}$ or $M_{y}=\bar{M}_{y}$,

$w_{0}=\bar{w}_{0}$ or $Q_{y}=\bar{Q}_{y}, \quad \theta_{z}=\bar{\theta}_{z}$ or $S_{y}=\bar{S}_{y}$,

$u_{0}^{*}=\bar{u}_{0}^{*}$ or $N_{x y}^{*}=\bar{N}_{x y}^{*}, \quad \theta_{x}^{*}=\bar{\theta}_{x}^{*}$ or $M_{x y}^{*}=\bar{M}_{x y}^{*}$,

$v_{0}^{*}=\bar{v}_{0}^{*}$ or $N_{y}^{*}=\bar{N}_{y}^{*}, \quad \theta_{y}^{*}=\bar{\theta}_{y}^{*}$ or $M_{y}^{*}=\bar{M}_{y}^{*}$,

$w_{0}^{*}=\bar{w}_{0}^{*}$ or $Q_{y}^{*}=\bar{Q}_{y}^{*}, \quad \theta_{z}^{*}=\bar{\theta}_{z}^{*}$ or $S_{y}^{*}=\bar{S}_{y}^{*}$,

where the stress resultants are defined by

$$
\left[\begin{array}{cc}
M_{x} & M_{x}^{*} \\
M_{y} & M_{y}^{*} \\
M_{z} & 0 \\
M_{x y} & M_{x y}^{*}
\end{array}\right]=\sum_{L=1}^{N L} \int_{z_{L}}^{z_{L+1}}\left\{\begin{array}{l}
\sigma_{x} \\
\sigma_{y} \\
\sigma_{z} \\
\tau_{x y}
\end{array}\right\}\left[\begin{array}{ll}
z & z^{3}
\end{array}\right] \mathrm{d} z
$$

$\left[\begin{array}{ll}Q_{x} & Q_{x}^{*} \\ Q_{y} & Q_{y}^{*}\end{array}\right]=\sum_{L=1}^{N L} \int_{z_{L}}^{z_{L+1}}\left\{\begin{array}{l}\tau_{x z} \\ \tau_{y z}\end{array}\right\}\left[\begin{array}{ll}1 & z^{2}\end{array}\right] \mathrm{d} z$,

$\left[\begin{array}{cc}N_{x} & N_{x}^{*} \\ N_{y} & N_{y}^{*} \\ N_{z} & N_{z}^{*} \\ N_{x y} & N_{x y}^{*}\end{array}\right]=\sum_{L=1}^{N L} \int_{z_{L}}^{z_{L+1}}\left\{\begin{array}{l}\sigma_{x} \\ \sigma_{y} \\ \sigma_{z} \\ \tau_{x y}\end{array}\right\}\left[\begin{array}{ll}1 & z^{2}\end{array}\right] \mathrm{d} z$

$\left[\begin{array}{ll}S_{x} & S_{x}^{*} \\ S_{y} & S_{y}^{*}\end{array}\right]=\sum_{L=1}^{N L} \int_{z_{L}}^{z_{L+1}}\left\{\begin{array}{l}\tau_{x z} \\ \tau_{y z}\end{array}\right\}\left[\begin{array}{ll}z & z^{3}\end{array}\right] \mathrm{d} z$.

The resultants in Eqs. (15)-(18) can be related to the total strains in Eq. (6) by the following equations:

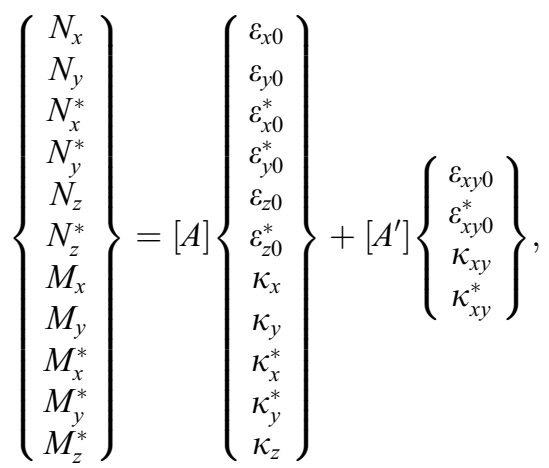

$\left\{\begin{array}{c}N_{x y} \\ N_{x y}^{*} \\ M_{x y} \\ M_{x y}^{*}\end{array}\right\}=\left[B^{\prime}\right]\left\{\begin{array}{c}\varepsilon_{x 0} \\ \varepsilon_{y 0} \\ \varepsilon_{x 0}^{*} \\ \varepsilon_{y 0}^{*} \\ \varepsilon_{z 0} \\ \varepsilon_{z 0}^{*} \\ \kappa_{x} \\ \kappa_{y} \\ \kappa_{x}^{*} \\ \kappa_{y}^{*} \\ \kappa_{z}\end{array}\right\}+[B]\left\{\begin{array}{c}\varepsilon_{x y 0} \\ \varepsilon_{x y 0}^{*} \\ \kappa_{x y} \\ \kappa_{x y}^{*}\end{array}\right\}$,

$\left\{\begin{array}{l}Q_{x} \\ Q_{x}^{*} \\ S_{x} \\ S_{x}^{*}\end{array}\right\}=[D]\left\{\begin{array}{c}\phi_{x} \\ \phi_{x}^{*} \\ \kappa_{x z} \\ \kappa_{x z}^{*}\end{array}\right\}+\left[D^{\prime}\right]\left\{\begin{array}{c}\phi_{y} \\ \phi_{y}^{*} \\ \kappa_{y z} \\ \kappa_{y z}^{*}\end{array}\right\}$,

$\left\{\begin{array}{c}Q_{y} \\ Q_{y}^{*} \\ S_{y} \\ S_{y}^{*}\end{array}\right\}=\left[E^{\prime}\right]\left\{\begin{array}{c}\phi_{x} \\ \phi_{x}^{*} \\ \kappa_{x z} \\ \kappa_{x z}^{*}\end{array}\right\}+[E]\left\{\begin{array}{c}\phi_{y} \\ \phi_{y}^{*} \\ \kappa_{y z} \\ \kappa_{y z}^{*}\end{array}\right\}$,

where the matrices $[A],\left[A^{\prime}\right],[B],\left[B^{\prime}\right],[D],\left[D^{\prime}\right],[E],\left[E^{\prime}\right]$ are the matrices of plate stiffnesses whose elements are defined in Appendix C.

\section{Analytical solutions}

Here the exact solutions of Eqs. (12)-(20) for crossply rectangular plates are considered. Assuming that the plate is simply supported in such a manner that normal displacement is admissible, but the tangential 
displacement is not, the following boundary conditions are appropriate:

At edges $x=0$ and $x=a$ :

$v_{0}=0, \quad w_{0}=0, \quad \theta_{y}=0, \quad \theta_{z}=0, \quad M_{x}=0$,

$v_{0}^{*}=0, \quad w_{0}^{*}=0, \quad \theta_{y}^{*}=0, \quad \theta_{z}^{*}=0, \quad M_{x}^{*}=0$,

$N_{x}=0, \quad N_{x}^{*}=0$.

At edges $y=0$ and $y=b$ :

$u_{0}=0, \quad w_{0}=0, \quad \theta_{x}=0, \quad \theta_{z}=0, \quad M_{y}=0$,

$u_{0}^{*}=0, \quad w_{0}^{*}=0, \quad \theta_{x}^{*}=0, \quad \theta_{z}^{*}=0, \quad M_{y}^{*}=0$,

$N_{y}=0, \quad N_{y}^{*}=0$.

Following Navier's solution procedure [2,3,30], the solution to the displacement variables satisfying the above boundary conditions can be expressed in the following forms:

$u_{0}=\sum_{m=1}^{\infty} \sum_{n=1}^{\infty} u_{0_{m n}} \cos \alpha x \sin \beta y$

$v_{0}=\sum_{m=1}^{\infty} \sum_{n=1}^{\infty} v_{0_{m n}} \sin \alpha x \cos \beta y$,

$w_{0}=\sum_{m=1}^{\infty} \sum_{n=1}^{\infty} w_{0_{m n}} \sin \alpha x \sin \beta y$,

$\theta_{x}=\sum_{m=1}^{\infty} \sum_{n=1}^{\infty} \theta_{x_{m n}} \cos \alpha x \sin \beta y$,

$\theta_{y}=\sum_{m=1}^{\infty} \sum_{n=1}^{\infty} \theta_{y_{m n}} \sin \alpha x \cos \beta y$,

$\theta_{z}=\sum_{m=1}^{\infty} \sum_{n=1}^{\infty} \theta_{z_{m n}} \sin \alpha x \sin \beta y$,

$u_{0}^{*}=\sum_{m=1}^{\infty} \sum_{n=1}^{\infty} u_{0_{m n}}^{*} \cos \alpha x \sin \beta y$,

$v_{0}^{*}=\sum_{m=1}^{\infty} \sum_{n=1}^{\infty} v_{0_{m n}}^{*} \sin \alpha x \cos \beta y$,

$w_{0}^{*}=\sum_{m=1}^{\infty} \sum_{n=1}^{\infty} w_{0_{m n}}^{*} \sin \alpha x \sin \beta y$,

$\theta_{x}^{*}=\sum_{m=1}^{\infty} \sum_{n=1}^{\infty} \theta_{x_{m n}}^{*} \cos \alpha x \sin \beta y$, $\theta_{y}^{*}=\sum_{m=1}^{\infty} \sum_{n=1}^{\infty} \theta_{y_{m n}}^{*} \sin \alpha x \cos \beta y$,

$\theta_{z}^{*}=\sum_{m=1}^{\infty} \sum_{n=1}^{\infty} \theta_{z_{m n}}^{*} \sin \alpha x \sin \beta y$,

and the loading term is expanded as

$p_{z}^{+}=\sum_{m=1}^{\infty} \sum_{n=1}^{\infty} p_{z_{m n}}^{+} \sin \alpha x \sin \beta y$,

where $\alpha=m \pi / a, \beta=n \pi / b$.

Substituting Eqs. (21)-(23) into Eq. (12) and collecting the coefficients one obtains

$[X]_{12 \times 12}\left\{\begin{array}{c}u_{0} \\ v_{0} \\ w_{0} \\ \theta_{x} \\ \theta_{y} \\ \theta_{z} \\ u_{0}^{*} \\ v_{0}^{*} \\ w_{0}^{*} \\ \theta_{x}^{*} \\ \theta_{y}^{*} \\ \theta_{z}^{*}\end{array}\right\}_{12 \times 1}=\left\{\begin{array}{c}0 \\ 0 \\ p_{z}^{+} \\ 0 \\ 0 \\ (h / 2)\left(p_{z}^{+}\right) \\ 0 \\ 0 \\ \left(h^{2} / 4\right)\left(p_{z}^{+}\right) \\ 0 \\ 0 \\ \left(h^{3} / 8\right)\left(p_{z}^{+}\right)\end{array}\right\}_{12 \times 1}$

for any fixed values of $m$ and $n$. The elements of coefficient matrix $[X]$ are given in Appendix D.

\section{Numerical results and discussion}

In this section, various numerical examples solved are described and discussed for establishing the accuracy of the various theories for the stress analysis of laminated composite and sandwich plates. The description of the various displacement models compared is given in Table 1. A shear correction factor of $5 / 6$ is used in computing results using Whitney-Pagano's theory. For all the problems a simply supported (diaphgram supported) plate is considered for the analysis. The transverse loading considered is sinusoidal. Results are obtained in closed form using Navier's solution technique for the above geometry and loading and the accuracy of the solution is established by comparing the

Table 1

Displacement models (shear deformation theories) compared

\begin{tabular}{lcccc}
\hline Source & Theory & Year (Ref.) & Degrees of freedom & $\begin{array}{l}\text { Transverse normal } \\
\text { deformation }\end{array}$ \\
\hline Present (Model-1) & & $1988([23])$ & 12 & Considered \\
Present (Model-2) & HSDT & $1988([22])$ & 9 & Not considered \\
Reddy (Model-3) & HSDT & $1984([15])$ & 5 & Not considered \\
Senthilnathan et al. (Model-4) & HSDT & $1987([16])$ & 4 & Not considered \\
Whitney-Pagano (Model-5) & HSDT & $1970([13])$ & 5 & Not considered \\
\hline
\end{tabular}


results with the exact elasticity solution wherever available in the literature.

The following sets of data are used in obtaining numerical results.

\section{Material 1 [28]}

$E_{1} / E_{2}=25, \quad E_{2}=E_{3}=10^{6} \mathrm{psi}(7 \mathrm{GPa})$,

$G_{12}=G_{13}=0.5 E_{2}, \quad G_{23}=0.2 E_{2}$,

$v_{12}=v_{23}=v_{13}=0.25$.

Material 2 [28]

Face sheets

$E_{1} / E_{2}=25, \quad E_{2}=E_{3}=10^{6}$,

$G_{12}=G_{13}=0.5 E_{2}, \quad G_{23}=0.2 E_{2}$,

$v_{12}=v_{23}=v_{13}=0.25$.
Core

$E_{1} / E_{3}=0.08, \quad E_{2} / E_{3}=0.08, \quad E_{3}=0.5 \times 10^{6}$,

$G_{13} / E_{3}=G_{23} / E_{3}=0.12, \quad v_{12}=0.25$,

$v_{23}=v_{13}=0.02$.

Material 3 [30]

Face sheets (graphite epoxy T300/934)

$E_{1}=19 \times 10^{6} \mathrm{psi}(131 \mathrm{GPa})$,

$E_{2}=1.5 \times 10^{6}$ psi $(10.34 \mathrm{GPa})$,

$E_{2}=E_{3}$,

$G_{12}=1 \times 10^{6}$ psi $(6.895 \mathrm{GPa})$,

$G_{13}=0.90 \times 10^{6} \mathrm{psi}(6.205 \mathrm{GPa})$,

$G_{23}=1 \times 10^{6}$ psi $(6.895 \mathrm{GPa})$,

$v_{12}=0.22, \quad v_{13}=0.22, \quad v_{23}=0.49$.

Table 2

Nondimensionalized deflections and stresses in a three-layer $\left(0^{\circ} / 90^{\circ} / 0^{\circ}\right)$ simply supported square laminate under sinusoidal transverse load

\begin{tabular}{|c|c|c|c|c|c|}
\hline$a / h$ & Theory & $\bar{w}$ & $\overline{\sigma_{x}}$ & ${\overline{\sigma_{y}}}^{\mathrm{a}}$ & $\overline{\bar{\tau}_{x y}}$ \\
\hline \multirow[t]{6}{*}{2} & Elasticity $^{\mathrm{b}}$ & - & 0.938 & 0.669 & 0.0859 \\
\hline & Present (Model-1) & 4.9147 & 1.1355 & 0.5356 & 0.0754 \\
\hline & Present (Model-2) & 5.2158 & 1.0912 & 0.6334 & 0.0803 \\
\hline & Model-3 & 5.1286 & 1.3112 & 0.5876 & 0.0889 \\
\hline & Model-4 & 4.3088 & 1.3460 & 0.1493 & 0.0532 \\
\hline & Model-5 & 5.2293 & 0.3597 & 0.7039 & 0.0488 \\
\hline \multirow[t]{6}{*}{4} & Elasticity $^{\mathrm{b}}$ & - & 0.755 & 0.556 & 0.0505 \\
\hline & Present (Model-1) & 1.8948 & 0.7648 & 0.4939 & 0.0487 \\
\hline & Present (Model-2) & 1.9261 & 0.7670 & 0.5079 & 0.0500 \\
\hline & Model-3 & 1.9218 & 0.7344 & 0.5028 & 0.0497 \\
\hline & Model-4 & 1.4852 & 0.7581 & 0.0902 & 0.0300 \\
\hline & Model-5 & 1.7758 & 0.4370 & 0.4774 & 0.0370 \\
\hline \multirow[t]{6}{*}{10} & Elasticity $^{\mathrm{b}}$ & - & 0.590 & 0.285 & 0.0289 \\
\hline & Present (Model-1) & 0.7151 & 0.5836 & 0.2705 & 0.0279 \\
\hline & Present (Model-2) & 0.7176 & 0.5847 & 0.2712 & 0.0281 \\
\hline & Model-3 & 0.7125 & 0.5684 & 0.2690 & 0.0277 \\
\hline & Model-4 & 0.6041 & 0.5747 & 0.1649 & 0.0227 \\
\hline & Model-5 & 0.6693 & 0.5134 & 0.2536 & 0.0252 \\
\hline \multirow[t]{6}{*}{20} & Elasticity $^{\mathrm{b}}$ & - & 0.552 & 0.210 & 0.0289 \\
\hline & Present (Model-1) & 0.5053 & 0.5504 & 0.2049 & 0.0231 \\
\hline & Present (Model-2) & 0.5058 & 0.5507 & 0.2050 & 0.0231 \\
\hline & Model-3 & 0.5041 & 0.5460 & 0.2043 & 0.0230 \\
\hline & Model-4 & 0.4746 & 0.5477 & 0.1759 & 0.0216 \\
\hline & Model-5 & 0.4921 & 0.5318 & 0.1997 & 0.0223 \\
\hline \multirow[t]{6}{*}{50} & Elasticity $^{\mathrm{b}}$ & - & 0.541 & 0.185 & 0.0216 \\
\hline & Present (Model-1) & 0.4432 & 0.5406 & 0.1838 & 0.0216 \\
\hline & Present (Model-2) & 0.4433 & 0.5406 & 0.1838 & 0.0216 \\
\hline & Model-3 & 0.4430 & 0.5399 & 0.1836 & 0.0216 \\
\hline & Model-4 & 0.4382 & 0.5401 & 0.1790 & 0.0213 \\
\hline & Model-5 & 0.4411 & 0.4480 & 0.1829 & 0.0215 \\
\hline \multirow[t]{6}{*}{100} & Elasticity $^{\mathrm{b}}$ & - & 0.539 & 0.181 & 0.0213 \\
\hline & Present (Model-1) & 0.4343 & 0.5392 & 0.1807 & 0.0214 \\
\hline & Present (Model-2) & 0.4343 & 0.5392 & 0.1807 & 0.0214 \\
\hline & Model-3 & 0.4342 & 0.5390 & 0.1806 & 0.0214 \\
\hline & Model-4 & 0.4330 & 0.5391 & 0.1795 & 0.0213 \\
\hline & Model-5 & 0.4337 & 0.5384 & 0.1804 & 0.0213 \\
\hline
\end{tabular}

${ }^{a}$ Max value occurs at $z= \pm h / 6$.

${ }^{\mathrm{b}}$ See [28]. 
Core (isotropic)

$E_{1}=E_{2}=E_{3}=2 G=1000 \mathrm{psi}\left(6.90 \times 10^{-3} \mathrm{GPa}\right)$,

$G_{12}=G_{13}=G_{23}=500$ psi $\left(3.45 \times 10^{-3} \mathrm{GPa}\right)$,

$v_{12}=v_{13}=v_{23}=0$.

Results reported in tables and plots are using the following nondimensional form:

$$
\begin{aligned}
& \bar{u}=u\left(\frac{100 h^{3} E_{2}}{P_{0} a^{4}}\right), \quad \bar{v}=v\left(\frac{100 h^{3} E_{2}}{P_{0} a^{4}}\right), \\
& \bar{w}=w\left(\frac{100 h^{3} E_{2}}{P_{0} a^{4}}\right), \quad \bar{\sigma}_{x}=\sigma_{x}\left(\frac{h^{2}}{P_{0} a^{2}}\right), \\
& \bar{\sigma}_{y}=\sigma_{y}\left(\frac{h^{2}}{P_{0} a^{2}}\right), \quad \bar{\tau}_{x y}=\tau_{x y}\left(\frac{h^{2}}{P_{0} a^{2}}\right) .
\end{aligned}
$$

Unless otherwise specified within the table(s) the locations (i.e. $x$-, $y$-, and $z$-coordinates) for maximum values of displacements, stresses and stress resultants for the present evaluations are as follows:

In-plane displacement $(u):(0, b / 2, \pm h / 2)$.

In-plane displacement $(v):(a / 2,0, \pm h / 2)$.

Transverse displacement $(w):(a / 2, b / 2,0)$.

In-plane normal stress $\left(\sigma_{x}\right):(a / 2, b / 2, \pm h / 2)$.

In-plane normal stress $\left(\sigma_{y}\right):(a / 2, b / 2, \pm h / 2)$.

In-plane shear stress $\left(\tau_{x y}\right):(0,0, \pm h / 2)$.

Example 1. A simply supported three-layered symmetric cross-ply $\left(0^{\circ} / 90^{\circ} / 0^{\circ}\right)$ square plate under sinusoidal transverse load is considered. The layers have equal thickness. Material set 1 is used. The numerical results of transverse displacement and in-plane stresses are given in Table 2. The numerical results of maximum in-plane stresses are compared with the exact elasticity solution given by [28]. The results clearly show that the values obtained using Model-2 and Model-3 are in close agreement for all $a / h$ ratios. For $a / h$ ratio equal to 2, Model-1 underpredicts deflection by $5.77 \%$, Model- 4 by $17.39 \%$ compared to the results of Model-2. Fig. 3 shows the through the thickness variation of in-plane displacement $\bar{u}$. It shows that the results obtained using Model-1, Model-2 and Model-3 are in good agreement whereas the values predicted by Model-4 and Model-5 differ from others considerably. Table 3 shows the percentage of error with respect to exact elasticity solution in computing the in-plane stresses. The results show that even at slenderness ratio as low as 2, Model-2 gives better accuracy compared to other displacement models. The accuracy of all models in predicting the in-plane stresses increases with increasing slenderness ratio. Figs. 4-6 shows the through the thickness variation of nondimensionalized in-plane stresses $\bar{\sigma}_{x}, \bar{\sigma}_{y}$ and $\bar{\tau}_{x y}$ for $a / h$ ratio equal to 10 . It shows that the stress values obtained using Model-1 and Model-2 are in excellent agreement.

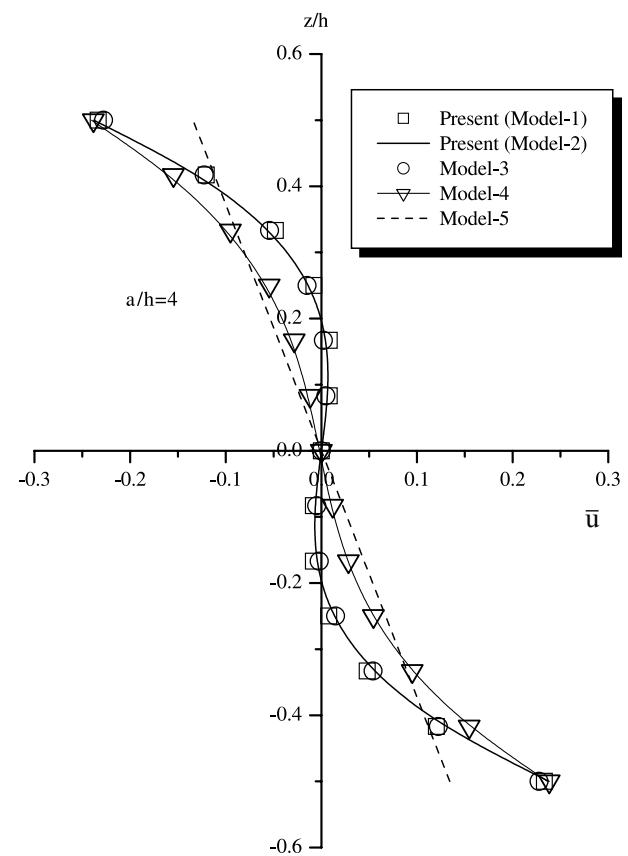

Fig. 3. Variation of nondimensionalized in-plane displacement $(\bar{u})$ through the thickness $(z / h)$ of a three-layer $\left(0^{\circ} / 90^{\circ} / 0^{\circ}\right)$ simply supported square plate under sinusoidal transverse load.

Table 3

Error $(\%)$ in a three-layer symmetric cross-ply $\left(0^{\circ} / 90^{\circ} / 0^{\circ}\right)$ laminate

\begin{tabular}{clrrr}
\hline$a / h$ & Theory & \multicolumn{1}{c}{$\bar{\sigma}_{x}$} & \multicolumn{1}{c}{$\bar{\sigma}_{y}$} & \multicolumn{1}{c}{$\bar{\tau}_{x y}$} \\
\hline \multirow{2}{*}{2} & Present (Model-1) & 21.05 & -19.94 & -12.22 \\
& Present (Model-2) & 16.33 & -5.32 & -6.52 \\
& Model-3 & 39.78 & -12.17 & 3.49 \\
& Model-4 & 43.49 & -77.68 & -38.07 \\
& Model-5 & -61.65 & 5.22 & -43.19 \\
\multirow{4}{*}{4} & Present (Model-1) & 1.30 & -11.17 & -3.56 \\
& Present (Model-2) & 1.59 & -8.65 & -0.99 \\
& Model-3 & -2.06 & -9.57 & -1.58 \\
& Model-4 & 0.41 & -83.78 & -40.59 \\
& Model-5 & -42.12 & -14.14 & -26.73 \\
& Present (Model-1) & -1.08 & -5.09 & -3.46 \\
& Present (Model-2) & -0.90 & -4.84 & -2.77 \\
& Model-3 & -3.66 & -5.61 & -4.15 \\
& Model-4 & -2.59 & -42.14 & -21.45 \\
& Model-5 & -12.98 & -11.02 & -12.80 \\
\hline
\end{tabular}

Example 2. A simply supported three-layered symmetric $\left(0^{\circ} /\right.$ core $\left./ 0^{\circ}\right)$ square sandwich plate with the thickness of each face sheet equal to $h / 10$ is considered. Material set 2 is used. The numerical results of transverse displacement and in-plane stresses for various aspect ratios $(a / h)$ are shown in Table 4 . The numerical results of maximum in-plane stresses are compared with exact elasticity solution given by [28]. Table 5 shows the percentage of error with respect to exact elasticity solution in computing the in-plane stresses for various $a / h$ ratios. The results show that the percentage 


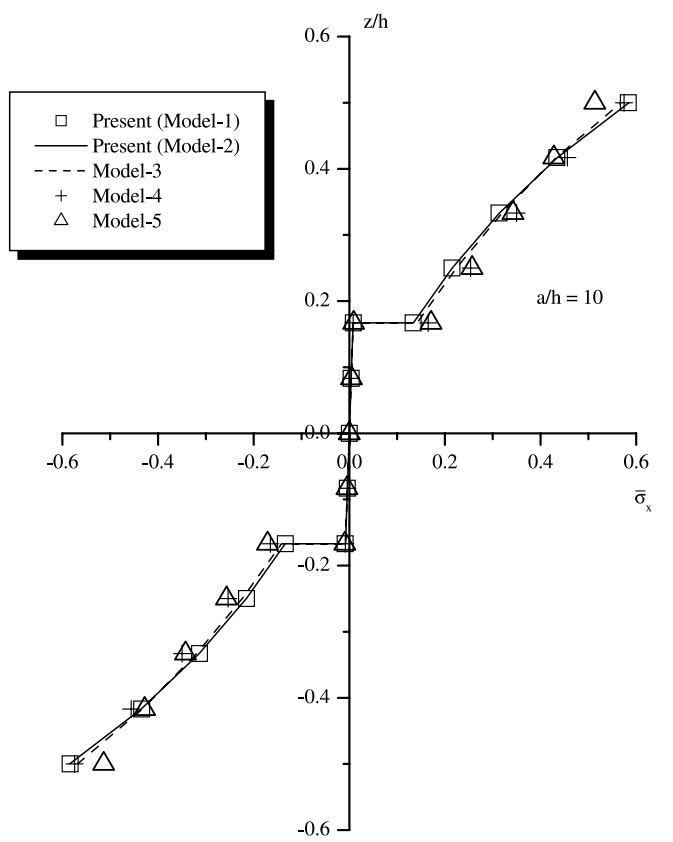

Fig. 4. Variation of nondimensionalized in-plane normal stress $\left(\bar{\sigma}_{x}\right)$ through the thickness $(z / h)$ of a three-layer $\left(0^{\circ} / 90^{\circ} / 0^{\circ}\right)$ simply supported square plate under sinusoidal transverse load.

of error got using the Model-4 and Model-5 is very large compared to other models and the percentage of error goes on reducing as the plate becomes thinner. For $a / h$ equal to 4,10 and 20 , Model-1 gives better estimate of in-plane stresses $\bar{\sigma}_{x}$ and $\bar{\sigma}_{y}$ whereas Model-2

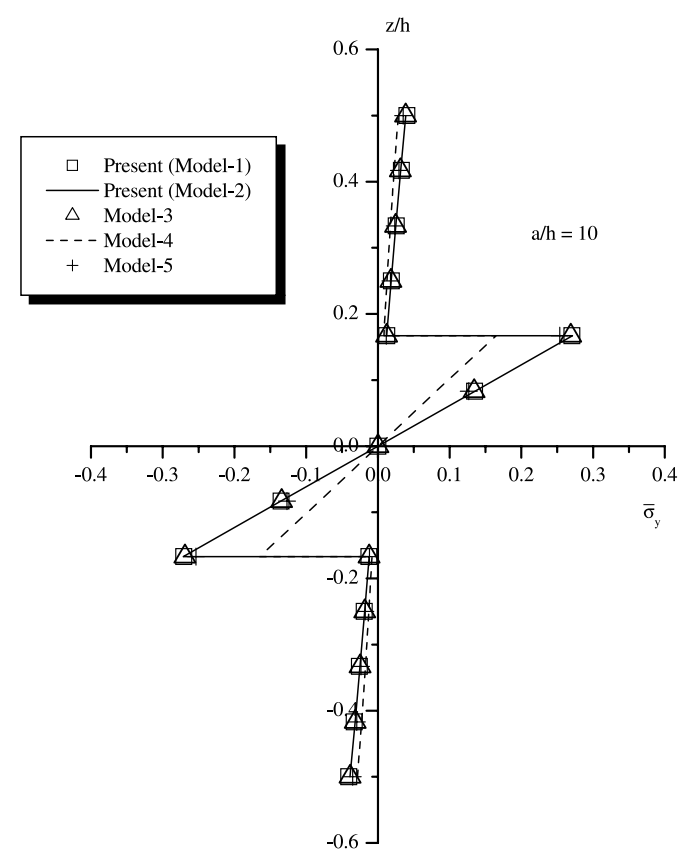

Fig. 5. Variation of nondimensionalized in-plane normal stress $\left(\bar{\sigma}_{y}\right)$ through the thickness $(z / h)$ of a three-layer $\left(0^{\circ} / 90^{\circ} / 0^{\circ}\right)$ simply supported square plate under sinusoidal transverse load.

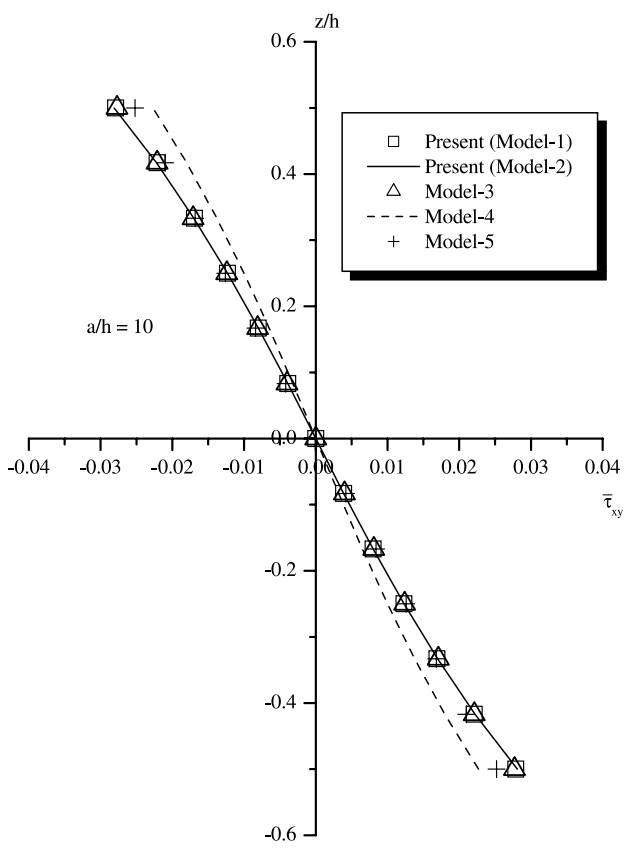

Fig. 6. Variation of nondimensionalized in-plane shear stress $\left(\bar{\tau}_{x y}\right)$ through the thickness $(z / h)$ of a three-layer $\left(0^{\circ} / 90^{\circ} / 0^{\circ}\right)$ simply supported square plate under sinusoidal transverse load.

predicts the in-plane shear stress more accurately than the other models for the above aspect ratios. For very thin laminate $(a / h=50$ and above) Model-2 gives better accurate results of all in-plane stresses as compared to other models. The percentage error in predicting the in-plane stresses $\bar{\sigma}_{x}$ and $\bar{\sigma}_{y}$ using Model-1 increases as the sandwich plate becomes thinner. The through the thickness variation of in-plane displacements $\bar{u}$ and $\bar{v}$ for $a / h$ ratio equal to 4 is shown in Figs. 7 and 8 . From the figures it can be seen that the values of in-plane displacements predicted by both Model-1 and Model-2 are same, there is a considerable difference in values of the above displacements predicted by other models.

Example 3. A simply supported two-layer $\left(0^{\circ} / 90^{\circ}\right)$ antisymmetric square laminate under sinusoidal transverse load is considered. The layers have equal thickness. Material set 1 is used. Numerical values of nondimensionalized transverse displacement and inplane stresses are shown in Table 6. Three-dimensional elasticity results are obtained using the method given by [28]. The percentage error with respect to threedimensional elasticity solution is given in Table 7. The results clearly indicate that the percentage error with respect to three-dimensional elasticity solution in predicting the transverse displacement and in-plane stresses is very much lesser in the case of Model-2 and the prediction of in-plane normal stresses $\bar{\sigma}_{x}, \bar{\sigma}_{y}$ by Model4 is very poor. 
Table 4

Nondimensionalized deflections and stresses in a three-layer simply supported square sandwich plate $\left(0^{\circ} /\right.$ core/ $\left.0^{\circ}\right)$ under sinusoidal transverse load

\begin{tabular}{|c|c|c|c|c|c|}
\hline$a / h$ & Theory & $\bar{w}$ & $\bar{\sigma}_{x}$ & $\bar{\sigma}_{y}$ & $\bar{\tau}_{x y}$ \\
\hline \multirow[t]{6}{*}{4} & Elasticity $^{\mathrm{a}}$ & - & 1.512 & 0.2533 & 0.1437 \\
\hline & Present (Model-1) & 7.0551 & 1.5137 & 0.2648 & 0.1379 \\
\hline & Present (Model-2) & 7.1539 & 1.5030 & 0.2391 & 0.1409 \\
\hline & Model-3 & 7.0873 & 1.4182 & 0.2365 & 0.1383 \\
\hline & Model-4 & 5.6205 & 1.5765 & 0.0780 & 0.0623 \\
\hline & Model-5 & 4.7666 & 0.8918 & 0.1562 & 0.0907 \\
\hline \multirow[t]{6}{*}{10} & Elasticity $^{\mathrm{a}}$ & - & 1.152 & 0.1099 & 0.0707 \\
\hline & Present (Model-1) & 2.0798 & 1.1523 & 0.1100 & 0.0685 \\
\hline & Present (Model-2) & 2.0848 & 1.1495 & 0.1042 & 0.0688 \\
\hline & Model-3 & 2.0629 & 1.1300 & 0.1030 & 0.0679 \\
\hline & Model-4 & 1.6458 & 1.1746 & 0.0581 & 0.0464 \\
\hline & Model-5 & 1.5604 & 1.0457 & 0.0798 & 0.0552 \\
\hline \multirow[t]{6}{*}{20} & Elasticity $^{\mathrm{a}}$ & - & 1.110 & 0.0700 & 0.0511 \\
\hline & Present (Model-1) & 1.1933 & 1.1110 & 0.0705 & 0.0504 \\
\hline & Present (Model-2) & 1.1939 & 1.1091 & 0.0682 & 0.0504 \\
\hline & Model-3 & 1.1876 & 1.1039 & 0.0679 & 0.0502 \\
\hline & Model-4 & 1.0704 & 1.1164 & 0.0552 & 0.0441 \\
\hline & Model-5 & 1.0524 & 1.0830 & 0.0612 & 0.0466 \\
\hline \multirow[t]{6}{*}{50} & Elasticity $^{\mathrm{a}}$ & - & 1.099 & 0.0569 & 0.0446 \\
\hline & Present (Model-1) & 0.9296 & 1.1005 & 0.0578 & 0.0445 \\
\hline & Present (Model-2) & 0.9294 & 1.0989 & 0.0566 & 0.0445 \\
\hline & Model-3 & 0.9284 & 1.0980 & 0.0565 & 0.0445 \\
\hline & Model-4 & 0.9090 & 1.1001 & 0.0545 & 0.0435 \\
\hline & Model-5 & 0.9063 & 1.0947 & 0.0554 & 0.0439 \\
\hline \multirow[t]{6}{*}{100} & Elasticity $^{\mathrm{a}}$ & - & 1.098 & 0.0550 & 0.0437 \\
\hline & Present (Model-1) & 0.8913 & 1.0990 & 0.0560 & 0.0436 \\
\hline & Present (Model-2) & 0.8910 & 1.0975 & 0.0549 & 0.0436 \\
\hline & Model-3 & 0.8908 & 1.0973 & 0.0549 & 0.0436 \\
\hline & Model-4 & 0.8859 & 1.0978 & 0.0543 & 0.0434 \\
\hline & Model-5 & 0.8852 & 1.0964 & 0.0546 & 0.0435 \\
\hline
\end{tabular}

${ }^{\mathrm{a}}$ See [28].

Example 4. In order to study the stretching-bending coupling effect, the analysis of a five-layer square sandwich plate $\left(0^{\circ} / 90^{\circ} /\right.$ core $\left./ 0^{\circ} / 90^{\circ}\right)$ with isotropic core and unbalanced cross-ply plates is presented. Material set 3 is used. The ratio of the thickness of the core $t_{\mathrm{c}}$ to thickness of the face sheet $t_{\mathrm{f}}$ considered is equal to 4 . Results are compared with the corresponding sandwich plate results with orthotropic faces. The numerical results of nondimensionalized transverse deflection, in-plane stresses of a five-layer sandwich plate with unbalanced cross-ply faces and a sandwich plate with orthotropic face sheets are given in Tables 8 and 9, respectively. The variation of nondimensionalized deflection ratio $\bar{w}_{\mathrm{c}} / \bar{w}_{0}$ (where $\bar{w}_{\mathrm{c}}$ is the nondimensionalized transverse deflection of sandwich plate with unbalanced cross-ply faces and $\bar{w}_{0}$ is the nondimensionalized transverse deflection of sandwich plate with orthotropic face sheets) with plate side-to-thickness ratio $(a / h)$ of a antisymmetric square sandwich plate under sinusoidal load is given in Fig. 9. From the figure it can be concluded that in the case of multilayer sandwich plate, the stretching-bending coupling effect is considerable in the case of thick plate and decreases the stiffness of the plate when higher order models are used. Irrespective of the thickness of the plate, the effect of coupling is always to increase the stiffness in the case of Model-5 and to reduce the stiffness of the plate in the case of Model-4. The higher order models, i.e., Model-1, Model-2 and Model3 , show that there is an increase in the stiffness when the plate thickness changes from thick to relatively thin, that is $a / h$ value changes from 2 to 50 . When the sandwich plate becomes very thin $(a / h=100)$, all the models show negligible coupling effect.

\section{Conclusion}

Analytical formulations and solutions to the static analysis of simply supported composite and sandwich plates hitherto not reported in the literature based on a higher order refined theory developed by the first author and already reported in the literature are presented. The displacement field of this theory takes into account both the transverse shear and normal deformations thus 
Table 5

Error $(\%)$ in a three-layered symmetric sandwich $\left(0^{\circ} /\right.$ core $\left./ 0^{\circ}\right)$ plate

\begin{tabular}{|c|c|c|c|c|}
\hline$a / h$ & Theory & $\bar{\sigma}_{x}$ & $\bar{\sigma}_{y}$ & $\bar{\tau}_{x y}$ \\
\hline 4 & $\begin{array}{l}\text { Present (Model-1) } \\
\text { Present (Model-2) } \\
\text { Model-3 } \\
\text { Model-4 } \\
\text { Model-5 }\end{array}$ & $\begin{array}{r}0.112 \\
-0.60 \\
-6.20 \\
4.26 \\
-41.02\end{array}$ & $\begin{array}{r}4.54 \\
-5.60 \\
-6.63 \\
-69.20 \\
-38.33\end{array}$ & $\begin{array}{r}-4.04 \\
-1.95 \\
-3.76 \\
-56.65 \\
-36.88\end{array}$ \\
\hline 10 & $\begin{array}{l}\text { Present (Model-1) } \\
\text { Present (Model-2) } \\
\text { Model-3 } \\
\text { Model-4 } \\
\text { Model-5 }\end{array}$ & $\begin{array}{r}0.026 \\
-0.22 \\
-1.91 \\
1.96 \\
-9.23\end{array}$ & $\begin{array}{c}0.091 \\
-5.19 \\
-6.28 \\
-47.13 \\
-27.39\end{array}$ & $\begin{array}{r}-3.11 \\
-2.69 \\
-3.96 \\
-34.37 \\
-21.92\end{array}$ \\
\hline 20 & $\begin{array}{l}\text { Present (Model-1) } \\
\text { Present (Model-2) } \\
\text { Model-3 } \\
\text { Model-4 } \\
\text { Model-5 }\end{array}$ & $\begin{array}{r}0.09 \\
-0.08 \\
-0.55 \\
0.58 \\
-2.43\end{array}$ & $\begin{array}{c}0.71 \\
-2.57 \\
-3.0 \\
-21.14 \\
-12.57\end{array}$ & $\begin{array}{r}-1.37 \\
-1.37 \\
-1.76 \\
-13.70 \\
-8.81\end{array}$ \\
\hline 50 & $\begin{array}{l}\text { Present (Model-1) } \\
\text { Present (Model-2) } \\
\text { Model-3 } \\
\text { Model-4 } \\
\text { Model-5 }\end{array}$ & $\begin{array}{c}0.136 \\
-0.091 \\
-0.091 \\
0.1 \\
-0.391\end{array}$ & $\begin{array}{l}1.58 \\
-0.527 \\
-0.703 \\
-4.22 \\
-2.64\end{array}$ & $\begin{array}{l}-0.224 \\
-0.224 \\
-0.224 \\
-2.47 \\
-1.57\end{array}$ \\
\hline 100 & $\begin{array}{l}\text { Present (Model-1) } \\
\text { Present (Model-2) } \\
\text { Model-3 } \\
\text { Model-4 } \\
\text { Model-5 }\end{array}$ & $\begin{array}{r}0.091 \\
-0.046 \\
-0.064 \\
-0.018 \\
-0.146\end{array}$ & $\begin{array}{l}1.82 \\
-0.182 \\
-0.182 \\
-1.27 \\
-0.727\end{array}$ & $\begin{array}{l}-0.229 \\
-0.229 \\
-0.229 \\
-0.686 \\
-0.458\end{array}$ \\
\hline
\end{tabular}

making it more accurate than the first order and other higher order theories considered. For laminated composite plates the solutions of the higher order refined

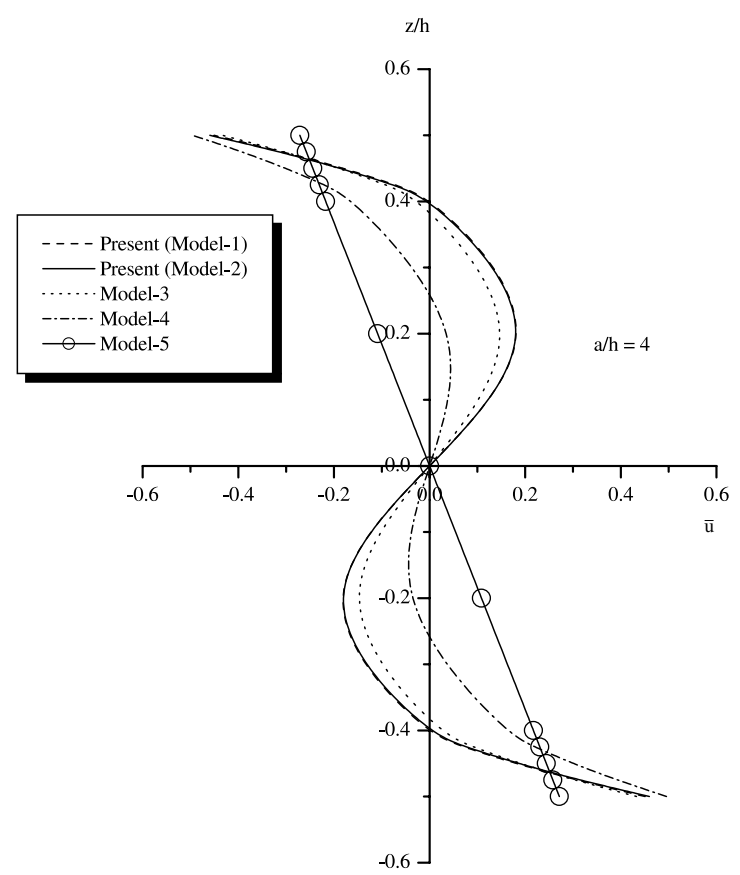

Fig. 7. Variation of nondimensionalized in-plane displacement $(\bar{u})$ through the thickness $(z / h)$ of a three-layer $\left(0^{\circ} /\right.$ core $\left./ 0^{\circ}\right)$ simply supported square plate under sinusoidal transverse load. theories (Model-1 and Model-2) are found to be in excellent agreement with the three-dimensional elasticity solutions and the percentage error with respect to $3 \mathrm{D}$

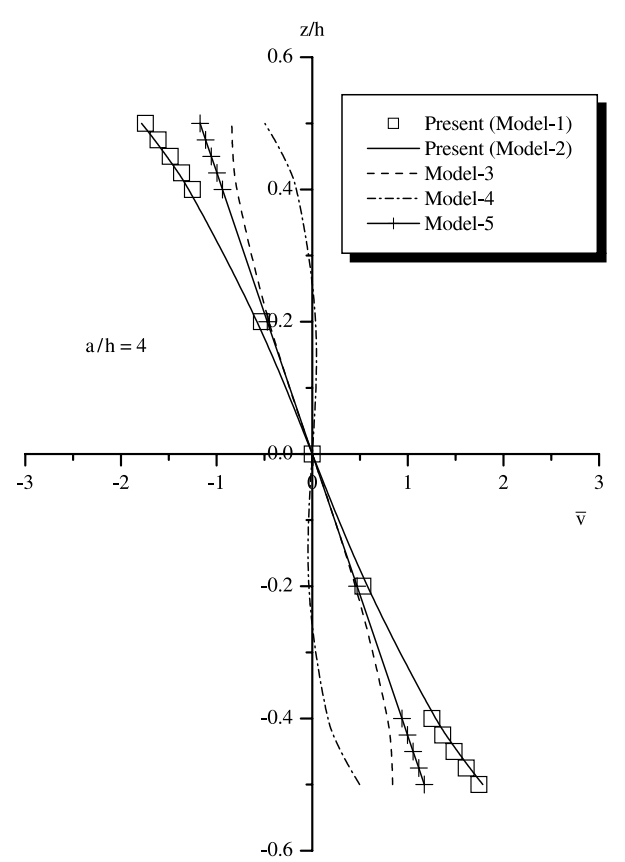

Fig. 8. Variation of nondimensionalized in-plane displacement $(\bar{v})$ through the thickness $(z / h)$ of a three-layer $\left(0^{\circ} /\right.$ core $\left./ 0^{\circ}\right)$ simply supported square plate under sinusoidal transverse load. 
Table 6

Nondimensionalized deflections and stresses in two-layer $\left(0^{\circ} / 90^{\circ}\right)$ simply supported square laminated plate under sinusoidal transverse load

\begin{tabular}{|c|c|c|c|c|c|}
\hline$a / h$ & Theory & $\bar{w}$ & $\bar{\sigma}_{x}$ & $\bar{\sigma}_{y}$ & $\bar{\tau}_{x y}$ \\
\hline \multirow[t]{6}{*}{2} & Elasticity $^{\mathrm{a}}$ & 4.9362 & -0.9070 & 1.4480 & -0.0964 \\
\hline & Present (Model-1) & 4.6558 & -0.8268 & 1.1946 & -0.0729 \\
\hline & Present (Model-2) & 4.9691 & -0.9535 & 0.9535 & -0.0758 \\
\hline & Model-3 & 4.5619 & -1.4277 & 1.4277 & -0.0719 \\
\hline & Model-4 & 4.5619 & -1.8199 & 1.8199 & -0.0719 \\
\hline & Model-5 & 5.4103 & -0.7151 & 0.7151 & -0.0527 \\
\hline \multirow[t]{6}{*}{5} & Elasticity $^{\mathrm{a}}$ & 1.7287 & -0.7723 & 0.8036 & -0.0586 \\
\hline & Present (Model-1) & 1.6800 & -0.7510 & 0.7720 & -0.0557 \\
\hline & Present (Model-2) & 1.7037 & -0.7662 & 0.7662 & -0.0572 \\
\hline & Model-3 & 1.6670 & -0.8385 & 0.8385 & -0.0558 \\
\hline & Model-4 & 1.6670 & -1.4133 & 1.4133 & -0.0558 \\
\hline & Model-5 & 1.7627 & -0.7151 & 0.7151 & -0.0527 \\
\hline \multirow[t]{6}{*}{10} & Elasticity $^{\mathrm{a}}$ & 1.2318 & -0.7317 & 0.7353 & -0.0540 \\
\hline & Present (Model-1) & 1.2192 & -0.7269 & 0.7273 & -0.0533 \\
\hline & Present (Model-2) & 1.2274 & -0.7286 & 0.7286 & -0.0539 \\
\hline & Model-3 & 1.2161 & -0.7468 & 0.7468 & -0.0533 \\
\hline & Model-4 & 1.2161 & -1.3500 & 1.3500 & -0.0533 \\
\hline & Model-5 & 1.2416 & -0.7151 & 0.7151 & -0.0527 \\
\hline \multirow[t]{6}{*}{20} & Elasticity $^{\mathrm{a}}$ & 1.1060 & -0.7200 & 0.7206 & -0.0529 \\
\hline & Present (Model-1) & 1.1025 & -0.7189 & 0.7186 & -0.0527 \\
\hline & Present (Model-2) & 1.1078 & -0.7185 & 0.7185 & -0.0530 \\
\hline & Model-3 & 1.1018 & -0.7235 & 0.7235 & -0.0527 \\
\hline & Model-4 & 1.1018 & -1.3340 & 1.3340 & -0.0527 \\
\hline & Model-5 & 1.1113 & -0.7151 & 0.7151 & -0.0527 \\
\hline \multirow[t]{6}{*}{100} & Elasticity $^{\mathrm{a}}$ & 1.0742 & -0.7219 & 0.7219 & -0.0529 \\
\hline & Present (Model-1) & 1.0651 & -0.7161 & 0.7161 & -0.0525 \\
\hline & Present (Model-2) & 1.0695 & -0.7152 & 0.7152 & -0.0527 \\
\hline & Model-3 & 1.0651 & -0.7161 & 0.7161 & -0.0525 \\
\hline & Model-4 & 1.0651 & -1.3288 & 1.3288 & -0.0525 \\
\hline & Model-5 & 1.0651 & -0.7151 & 0.7151 & -0.0527 \\
\hline
\end{tabular}

${ }^{\mathrm{a}}$ See [28].

Table 7

Percentage error in a two-layer $\left(0^{\circ} / 90^{\circ}\right)$ cross-ply laminate

\begin{tabular}{|c|c|c|c|c|c|}
\hline$a / h$ & Theory & $\bar{w}$ & $\bar{\sigma}_{x}$ & $\bar{\sigma}_{y}$ & $\bar{\tau}_{x y}$ \\
\hline \multirow[t]{5}{*}{5} & Present (Model-1) & -2.82 & -1.98 & -3.93 & 4.95 \\
\hline & Present (Model-2) & -1.45 & -0.79 & -4.65 & -2.39 \\
\hline & Model-3 & -3.57 & 8.57 & 4.34 & -4.78 \\
\hline & Model-4 & -3.57 & 83.00 & 75.87 & -4.78 \\
\hline & Model-5 & 1.97 & -7.41 & -11.01 & -10.07 \\
\hline \multirow[t]{5}{*}{10} & Present (Model-1) & -1.02 & -0.66 & -1.09 & -1.30 \\
\hline & Present (Model-2) & -0.36 & -0.42 & -0.91 & -0.19 \\
\hline & Model-3 & -1.27 & 2.06 & 1.56 & -1.30 \\
\hline & Model-4 & -1.27 & 84.50 & 83.60 & -1.30 \\
\hline & Model-5 & 0.80 & -2.27 & -2.02 & -2.41 \\
\hline \multirow[t]{5}{*}{100} & Present (Model-1) & -0.85 & -0.80 & -0.80 & -0.76 \\
\hline & Present (Model-2) & -0.44 & -0.93 & -0.93 & -0.38 \\
\hline & Model-3 & -0.85 & -0.80 & -0.80 & -0.76 \\
\hline & Model-4 & -0.85 & 84.07 & 84.07 & -0.76 \\
\hline & Model-5 & -0.85 & -0.94 & -0.94 & -0.38 \\
\hline
\end{tabular}

elasticity solutions is very much less compared to other shear deformation theories used for comparison in this study. For sandwich plates the results of Kant-Manju- natha and Pandya-Kant theories are in good agreement whereas the error is quite considerable when the first order theory and the theories of Reddy and Senthilna- 
Table 8

Nondimensionalized deflections and stresses in a five-layer $\left(0^{\circ} / 90^{\circ} / \mathrm{core} / 0^{\circ} / 90^{\circ}\right)$ simply supported square sandwich plate under sinusoidal transverse load

\begin{tabular}{clrrrr}
\hline$a / h$ & Theory & $\bar{w}_{\mathrm{c}}$ & $\bar{\sigma}_{x}$ & $\bar{\sigma}_{y}$ & $\bar{\tau}_{x y}$ \\
\hline 2 & Present (Model-1) & 43.2468 & 2.6494 & 4.5305 & -0.7688 \\
& Present (Model-2) & 44.9032 & -3.5349 & 3.5349 & 0.5515 \\
& Model-3 & 30.2539 & -2.0269 & 2.0269 & 0.2959 \\
& Model-4 & 30.2539 & -2.8710 & 2.8709 & 0.2959 \\
& Model-5 & 8.6438 & -0.6199 & 0.6199 & 0.0693 \\
4 & Present (Model-1) & 14.1627 & -1.6445 & 1.4931 & 0.2031 \\
& Present (Model-2) & 14.3440 & -1.5328 & 1.5328 & 0.2196 \\
& Model-3 & 8.7941 & -0.9937 & 0.9937 & 0.1291 \\
& Model-4 & 8.7941 & -1.2525 & 1.2524 & 0.1291 \\
& Model-5 & 2.9509 & -0.6199 & 0.6199 & 0.0693 \\
& Present (Model-1) & 3.3032 & -0.8104 & 0.7606 & 0.0946 \\
& Present (Model-2) & 3.3197 & -0.7771 & 0.7771 & 0.0951 \\
& Model-3 & 2.3075 & -0.6815 & 0.6815 & 0.0787 \\
& Model-4 & 2.3075 & -0.7634 & 0.7631 & 0.0787 \\
& Model-5 & 1.3570 & -0.6200 & 0.6200 & 0.0693 \\
& Present (Model-1) & 1.0697 & -0.6231 & 0.6226 & 0.0691 \\
& Present (Model-2) & 1.0763 & -0.6216 & 0.6216 & 0.0696 \\
& Model-3 & 1.0595 & -0.6214 & 0.6214 & 0.0690 \\
& Model-4 & 1.0595 & -0.6692 & 0.6691 & 0.0690 \\
\end{tabular}

than et al. are used. The main aim of this entire investigation is to bring out clearly the accuracy of the various shear deformation theories in predicting the stresses so that the claims made by various investigators regarding the supremacy of their models are put to rest.

\section{Appendix A. Coefficients of $[C]$ matrix}

$C_{11}=\frac{E_{1}\left(1-v_{23} v_{32}\right)}{\Delta}, \quad C_{12}=\frac{E_{1}\left(v_{21}+v_{31} v_{23}\right)}{\Delta}$

Table 9

Nondimensionalized deflections and stresses in a simply supported square sandwich plate with orthotropic face sheets under sinusoidal transverse loads

\begin{tabular}{rlrlll}
\hline$a / h$ & Theory & $\bar{w}_{0}$ & $\bar{\sigma}_{x}$ & $\bar{\sigma}_{y}$ & $\bar{\tau}_{x y}$ \\
\hline 2 & Present (Model-1) & 37.8550 & -2.3560 & 0.3752 & 0.3172 \\
& Present (Model-2) & 39.0218 & -4.0665 & 0.5310 & 0.5184 \\
& Model-3 & 29.4799 & -2.4689 & 0.3922 & 0.3583 \\
& Model-4 & 28.3619 & -2.6684 & 0.2526 & 0.2750 \\
& Model-5 & 9.7795 & -0.3814 & 0.1943 & 0.1440 \\
4 & Present (Model-1) & 14.3218 & -1.7931 & 0.2128 & 0.2702 \\
& Present (Model-2) & 14.4949 & -1.8204 & 0.3077 & 0.2783 \\
& Model-3 & 9.6703 & -1.0558 & 0.2358 & 0.1978 \\
& Model-4 & 8.6085 & -1.2193 & 0.1154 & 0.1257 \\
& Model-5 & 3.5348 & -0.5176 & 0.1308 & 0.1077 \\
& Present (Model-1) & 3.8714 & -0.8536 & 0.1372 & 0.1363 \\
& Present (Model-2) & 3.8899 & -0.8344 & 0.1527 & 0.1352 \\
& Model-3 & 2.7265 & -0.6913 & 0.1198 & 0.1069 \\
& Model-4 & 2.2887 & -0.7558 & 0.0715 & 0.0779 \\
& Model-5 & 1.4854 & -0.6301 & 0.0784 & 0.0777 \\
& Present (Model-1) & 1.0740 & -0.6657 & 0.0641 & 0.0694 \\
& Present (Model-2) & 1.0806 & -0.6647 & 0.0642 & 0.0699 \\
& Model-3 & 1.0591 & -0.6642 & 0.0636 & 0.0689 \\
& Model-4 & 1.0531 & -0.6651 & 0.0630 & 0.0690 \\
\end{tabular}




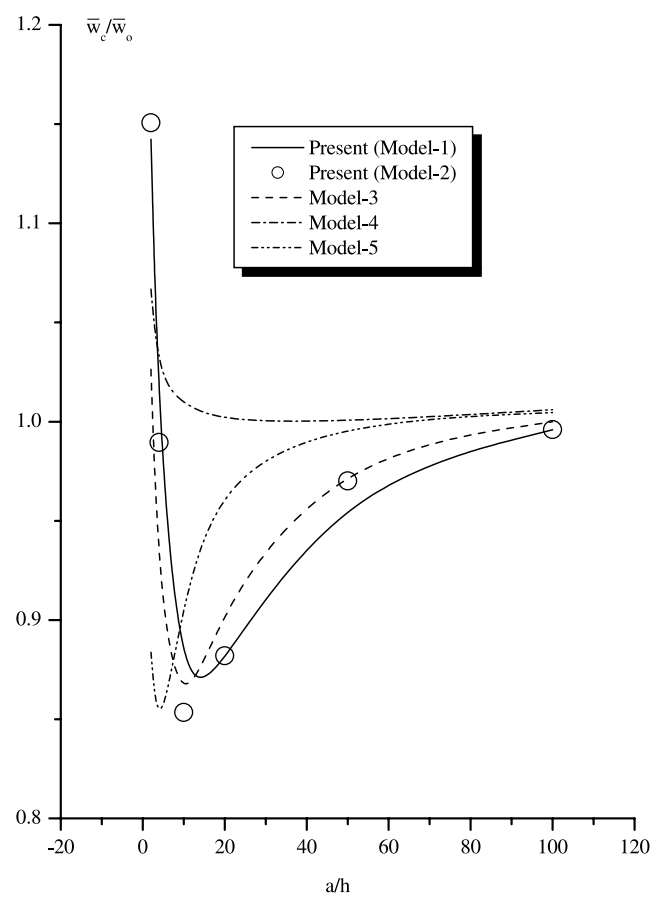

Fig. 9. Nondimensionalized deflection ratio $\left(\bar{w}_{\mathrm{c}} / \bar{w}_{0}\right)$ as a function of plate side-to-thickness ratio $(a / h)$ of a five-layer $\left(0 \% / 90 \%\right.$ core $\left./ 0^{\circ} / 90^{\circ}\right)$ simply supported square sandwich plate under sinusoidal transverse load.

$C_{13}=\frac{E_{1}\left(v_{31}+v_{21} v_{32}\right)}{\Delta}, \quad C_{22}=\frac{E_{2}\left(1-v_{13} v_{31}\right)}{\Delta}$,

$C_{23}=\frac{E_{2}\left(v_{32}+v_{12} v_{31}\right)}{\Delta}, \quad C_{33}=\frac{E_{3}\left(1-v_{12} v_{21}\right)}{\Delta}$,

$C_{44}=G_{12}, \quad C_{55}=G_{23}, \quad C_{66}=G_{13}$,

where

$\Delta=\left(1-v_{12} v_{21}-v_{23} v_{32}-v_{31} v_{13}-2 v_{12} v_{23} v_{31}\right)$

and

$\varepsilon_{1}=\frac{\sigma_{1}}{E_{1}}-v_{21} \frac{\sigma_{2}}{E_{2}}-v_{31} \frac{\sigma_{3}}{E_{3}}$,

$$
\begin{aligned}
& \varepsilon_{2}=\frac{\sigma_{2}}{E_{2}}-v_{32} \frac{\sigma_{3}}{E_{3}}-v_{12} \frac{\sigma_{1}}{E_{1}}, \\
& \varepsilon_{3}=\frac{\sigma_{3}}{E_{3}}-v_{13} \frac{\sigma_{1}}{E_{1}}-v_{23} \frac{\sigma_{2}}{E_{2}}, \\
& \gamma_{12}=\frac{\tau_{12}}{G_{12}}, \quad \gamma_{23}=\frac{\tau_{23}}{G_{23}}, \quad \gamma_{13}=\frac{\tau_{13}}{G_{13}}, \\
& \frac{v_{12}}{E_{1}}=\frac{v_{21}}{E_{2}}, \quad \frac{v_{31}}{E_{3}}=\frac{v_{13}}{E_{1}}, \quad \frac{v_{32}}{E_{3}}=\frac{v_{23}}{E_{2}} .
\end{aligned}
$$

\section{Appendix B. Coefficients of $[Q]$ matrix}

$$
\begin{aligned}
& Q_{11}=C_{11} c^{4}+2\left(C_{12}+2 C_{44}\right) s^{2} c^{2}+C_{22} s^{4}, \\
& Q_{12}=C_{12}\left(c^{4}+s^{4}\right)+\left(C_{11}+C_{22}-4 C_{44}\right) s^{2} c^{2}, \\
& Q_{13}=C_{13} c^{2}+C_{23} s^{2}, \\
& Q_{14}=\left(C_{11}-C_{12}-2 C_{44}\right) s c^{3}+\left(C_{12}-C_{22}+2 C_{44}\right) c s^{3}, \\
& Q_{22}=C_{11} s^{4}+C_{22} c^{4}+\left(2 C_{12}+4 C_{44}\right) s^{2} c^{2}, \\
& Q_{23}=C_{13} s^{2}+C_{23} c^{2}, \\
& Q_{24}=\left(C_{11}-C_{12}-2 C_{44}\right) s^{3} c+\left(C_{12}-C_{22}+2 C_{44}\right) c^{3} s, \\
& Q_{33}=C_{33}, \\
& Q_{34}=\left(C_{31}-C_{32}\right) s c, \\
& Q_{44}=\left(C_{11}-2 C_{12}+C_{22}-2 C_{44}\right) c^{2} s^{2}+C_{44}\left(c^{4}+s^{4}\right), \\
& Q_{55}=C_{55} c^{2}+C_{66} s^{2}, \\
& Q_{56}=\left(C_{66}-C_{55}\right) c s, \\
& Q_{66}=C_{55} s^{2}+C_{66} c^{2}
\end{aligned}
$$

and

$Q_{i j}=Q_{j i}, \quad i, j=1$ to 6,

where

$c=\cos \alpha \quad$ and $\quad s=\sin \alpha$.

Appendix C. Elements of $[\boldsymbol{A}],\left[\boldsymbol{A}^{\prime}\right],[\boldsymbol{B}],\left[\boldsymbol{B}^{\prime}\right],[\boldsymbol{D}],\left[\boldsymbol{D}^{\prime}\right],[\boldsymbol{E}]$, $\left[E^{\prime}\right]$ matrices

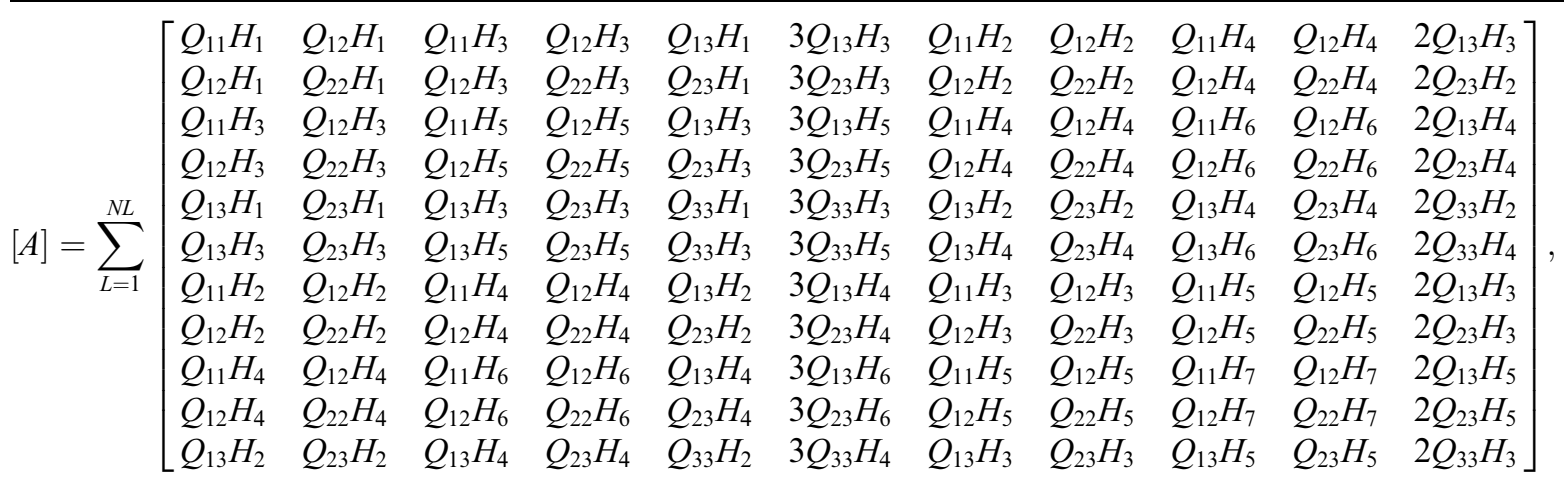




$$
\begin{array}{r}
{[B]=\sum_{L=1}^{N L}\left[\begin{array}{llll}
Q_{44} H_{1} & Q_{44} H_{3} & Q_{44} H_{2} & Q_{44} H_{4} \\
Q_{44} H_{3} & Q_{44} H_{5} & Q_{44} H_{4} & Q_{44} H_{6} \\
Q_{44} H_{2} & Q_{44} H_{4} & Q_{44} H_{3} & Q_{44} H_{5} \\
Q_{44} H_{4} & Q_{44} H_{6} & Q_{44} H_{5} & Q_{44} H_{7}
\end{array}\right],} \\
{\left[A^{\prime}\right]=\sum_{L=1}^{N L}\left[\begin{array}{llll}
Q_{14} H_{1} & Q_{14} H_{3} & Q_{14} H_{2} & Q_{14} H_{4} \\
Q_{24} H_{1} & Q_{24} H_{3} & Q_{24} H_{2} & Q_{24} H_{4} \\
Q_{14} H_{3} & Q_{14} H_{5} & Q_{14} H_{4} & Q_{14} H_{6} \\
Q_{24} H_{3} & Q_{24} H_{5} & Q_{24} H_{4} & Q_{24} H_{6} \\
Q_{34} H_{1} & Q_{34} H_{3} & Q_{34} H_{2} & Q_{34} H_{4} \\
Q_{34} H_{3} & Q_{34} H_{5} & Q_{34} H_{4} & Q_{34} H_{6} \\
Q_{14} H_{2} & Q_{14} H_{4} & Q_{14} H_{3} & Q_{14} H_{5} \\
Q_{24} H_{2} & Q_{24} H_{4} & Q_{24} H_{3} & Q_{24} H_{5} \\
Q_{14} H_{4} & Q_{14} H_{6} & Q_{14} H_{5} & Q_{14} H_{7} \\
Q_{24} H_{4} & Q_{24} H_{6} & Q_{24} H_{5} & Q_{24} H_{7} \\
Q_{34} H_{2} & Q_{34} H_{4} & Q_{34} H_{3} & Q_{34} H_{5}
\end{array}\right],}
\end{array}
$$

$$
\begin{aligned}
& X_{2,2}=A_{2,2} \beta^{2}+B_{1,2} \alpha^{2}, \quad X_{2,3}=0, \\
& X_{2,4}=A_{2,7} \alpha \beta+B_{1,5} \alpha \beta, \\
& X_{2,5}=A_{2,8} \beta^{2}+B_{1,6} \alpha^{2}, \quad X_{2,6}=-A_{2,5} \beta, \\
& X_{2,7}=A_{2,3} \alpha \beta+B_{1,3} \alpha \beta, \quad X_{2,8}=A_{2,4} \beta^{2}+B_{1,4} \alpha^{2}, \\
& X_{2,9}=-A_{2,11} \beta, \quad X_{2,10}=A_{2,9} \alpha \beta+B_{1,7} \alpha \beta, \\
& X_{2,11}=A_{2,10} \beta^{2}+B_{1,8} \alpha^{2}, \quad X_{2,12}=-A_{2,6} \beta, \\
& X_{3,3}=D_{1,2} \alpha^{2}+E_{1,2} \beta^{2}, \quad X_{3,4}=D_{1,1} \alpha, \\
& X_{3,5}=E_{1,1} \beta, \quad X_{3,6}=D_{1,6} \alpha^{2}+E_{1,6} \beta^{2}, \\
& X_{3,7}=D_{1,5} \alpha, \quad X_{3,8}=E_{1,5} \beta, \\
& X_{3,9}=D_{1,4} \alpha^{2}+E_{1,4} \beta^{2}, \quad X_{3,10}=D_{1,3} \alpha, \\
& X_{3,11}=E_{1,3} \beta, \quad X_{3,12}=D_{1,7} \alpha^{2}+E_{1,7} \beta^{2}, \\
& X_{4,4}=A_{7,7} \alpha^{2}+B_{3,5} \beta^{2}+D_{1,1}, \quad X_{4,5}=A_{7,8} \alpha \beta+B_{3,6} \alpha \beta,
\end{aligned}
$$

$\left[B^{\prime}\right]=\sum_{L=1}^{N L}\left[\begin{array}{lllllllllll}Q_{14} H_{1} & Q_{24} H_{1} & Q_{14} H_{3} & Q_{24} H_{3} & Q_{34} H_{1} & 3 Q_{34} H_{3} & Q_{14} H_{2} & Q_{24} H_{2} & Q_{14} H_{4} & Q_{24} H_{4} & 2 Q_{34} H_{2} \\ Q_{14} H_{3} & Q_{24} H_{3} & Q_{14} H_{5} & Q_{24} H_{5} & Q_{34} H_{3} & 3 Q_{34} H_{5} & Q_{14} H_{4} & Q_{24} H_{4} & Q_{14} H_{6} & Q_{24} H_{6} & 2 Q_{34} H_{4} \\ Q_{14} H_{2} & Q_{24} H_{2} & Q_{14} H_{4} & Q_{24} H_{4} & Q_{34} H_{2} & 3 Q_{34} H_{4} & Q_{14} H_{3} & Q_{24} H_{3} & Q_{14} H_{5} & Q_{24} H_{5} & 2 Q_{34} H_{3} \\ Q_{14} H_{4} & Q_{24} H_{4} & Q_{14} H_{6} & Q_{24} H_{6} & Q_{34} H_{4} & 3 Q_{34} H_{6} & Q_{14} H_{5} & Q_{24} H_{5} & Q_{14} H_{7} & Q_{24} H_{7} & 2 Q_{34} H_{5}\end{array}\right]$,

$$
\begin{gathered}
{[D]=\sum_{L=1}^{N L}\left[\begin{array}{llll}
Q_{66} H_{1} & Q_{66} H_{3} & Q_{66} H_{2} & Q_{66} H_{4} \\
Q_{66} H_{3} & Q_{66} H_{5} & Q_{66} H_{4} & Q_{66} H_{6} \\
Q_{66} H_{2} & Q_{66} H_{4} & Q_{66} H_{3} & Q_{66} H_{5} \\
Q_{66} H_{4} & Q_{66} H_{6} & Q_{66} H_{5} & Q_{66} H_{7}
\end{array}\right],} \\
{\left[D^{\prime}\right]=\sum_{L=1}^{N L}\left[\begin{array}{llll}
Q_{56} H_{1} & Q_{56} H_{3} & Q_{56} H_{2} & Q_{56} H_{4} \\
Q_{56} H_{3} & Q_{56} H_{5} & Q_{56} H_{4} & Q_{56} H_{6} \\
Q_{56} H_{2} & Q_{56} H_{4} & Q_{56} H_{3} & Q_{56} H_{5} \\
Q_{56} H_{4} & Q_{56} H_{6} & Q_{56} H_{5} & Q_{56} H_{7}
\end{array}\right],} \\
{[E]=\sum_{L=1}^{N L}\left[\begin{array}{llll}
Q_{55} H_{1} & Q_{55} H_{3} & Q_{55} H_{2} & Q_{55} H_{4} \\
Q_{55} H_{3} & Q_{55} H_{5} & Q_{55} H_{4} & Q_{55} H_{6} \\
Q_{55} H_{2} & Q_{55} H_{4} & Q_{55} H_{3} & Q_{55} H_{5} \\
Q_{55} H_{4} & Q_{55} H_{6} & Q_{55} H_{5} & Q_{55} H_{7}
\end{array}\right],} \\
{\left[\begin{array}{llll}
Q_{56} H_{1} & Q_{56} H_{3} & Q_{56} H_{2} & Q_{56} H_{4} \\
Q_{56} H_{3} & Q_{56} H_{5} & Q_{56} H_{4} & Q_{56} H_{6} \\
Q_{56} H_{2} & Q_{56} H_{4} & Q_{56} H_{3} & Q_{56} H_{5} \\
Q_{56} H_{4} & Q_{56} H_{6} & Q_{56} H_{5} & Q_{56} H_{7}
\end{array}\right] .}
\end{gathered}
$$

\section{Appendix D. Coefficients of matrix $[X]$}

$$
\begin{aligned}
& X_{1,1}=A_{1,1} \alpha^{2}+B_{1,1} \beta^{2}, \quad X_{1,2}=A_{1,2} \alpha \beta+B_{1,2} \alpha \beta, \\
& X_{1,3}=0, \quad X_{1,4}=A_{1,7} \alpha^{2}+B_{1,5} \beta^{2}, \\
& X_{1,5}=A_{1,8} \alpha \beta+B_{1,6} \alpha \beta, \\
& X_{1,6}=-A_{1,5} \alpha, \quad X_{1,7}=A_{1,3} \alpha^{2}+B_{1,3} \beta^{2}, \\
& X_{1,8}=A_{1,4} \alpha \beta+B_{1,4} \alpha \beta, \quad X_{1,9}=-A_{1,11} \alpha, \\
& X_{1,10}=A_{1,9} \alpha^{2}+B_{1,7} \beta^{2}, \quad X_{1,11}=A_{1,10} \alpha \beta+B_{1,8} \alpha \beta, \\
& X_{1,12}=-A_{1,6} \alpha,
\end{aligned}
$$

$$
\begin{aligned}
& X_{4,6}=-A_{7,5} \alpha+D_{1,6} \alpha, \quad X_{4,7}=A_{7,3} \alpha^{2}+B_{3,3} \beta^{2}+D_{1,5}, \\
& X_{4,8}=A_{7,4} \alpha \beta+B_{3,4} \alpha \beta, \quad X_{4,9}=-A_{7,11} \alpha+D_{1,4} \alpha, \\
& X_{4,10}=A_{7,9} \alpha^{2}+B_{3,7} \beta^{2}+D_{1,3}, \quad X_{4,11}=A_{7,10} \alpha \beta+B_{3,8} \alpha \beta, \\
& X_{4,12}=-A_{7,6} \alpha+D_{1,7} \alpha, \\
& X_{5,5}=A_{8,8} \beta^{2}+B_{3,6} \alpha^{2}+E_{1,1}, \quad X_{5,6}=-A_{8,5} \beta+E_{1,6} \beta, \\
& X_{5,7}=A_{8,3} \alpha \beta+B_{3,3} \alpha \beta, \quad X_{5,8}=A_{8,4} \beta^{2}+B_{3,4} \alpha^{2}+E_{1,5}, \\
& X_{5,9}=-A_{8,11} \beta+E_{1,4} \beta, \quad X_{5,10}=A_{8,9} \alpha \beta+B_{3,7} \alpha \beta, \\
& X_{5,11}=A_{8,10} \beta^{2}+B_{3,8} \alpha^{2}+E_{1,3}, \quad X_{5,12}=-A_{8,6} \beta+E_{1,7} \beta, \\
& X_{6,6}=D_{3,6} \alpha^{2}+E_{3,6} \beta^{2}+A_{5,5}, \quad X_{6,7}=D_{3,5} \alpha-A_{5,3} \alpha, \\
& X_{6,8}=E_{3,5} \beta-A_{5,4} \beta, \quad X_{6,9}=D_{3,4} \alpha^{2}+E_{3,4} \beta^{2}+A_{5,11}, \\
& X_{6,10}=D_{3,3} \alpha-A_{5,9} \alpha, \quad X_{6,11}=E_{3,3} \beta-A_{5,10} \beta, \\
& X_{6,12}=D_{3,7} \alpha^{2}+E_{3,7} \beta^{2}+A_{5,6}, \\
& X_{7,7}=A_{3,3} \alpha^{2}+B_{2,3} \beta^{2}+2 D_{3,5}, \quad X_{7,8}=A_{3,4} \alpha \beta+B_{2,4} \alpha \beta, \\
& X_{7,9}=-A_{3,11} \alpha+2 D_{3,4} \alpha, \quad X_{7,10}=A_{3,9} \alpha^{2}+B_{2,7} \beta^{2}+2 D_{3,3}, \\
& X_{7,11}=A_{3,10} \alpha \beta+B_{2,8} \alpha \beta, \quad X_{7,12}=-A_{3,6} \alpha+2 D_{3,7} \alpha, \\
& X_{8,8}=A_{4,4} \beta^{2}+B_{2,4} \alpha^{2}+2 E_{3,5}, \quad X_{8,9}=-A_{4,11} \beta+2 E_{3,4} \beta, \\
& X_{8,10}=A_{4,9} \alpha \beta+B_{2,7} \alpha \beta, \quad X_{8,11}=A_{4,10} \beta^{2}+B_{2,8} \alpha^{2}+2 E_{3,3}, \\
& X_{8,12}=-A_{4,6} \beta+2 E_{3,7} \beta, \\
& X_{9,9}=D_{2,4} \alpha^{2}+E_{2,4} \beta^{2}+2 A_{11,11}, \quad X_{9,10}=D_{2,3} \alpha-2 A_{11,9} \alpha, \\
& X_{9,11}=E_{2,3} \beta-2 A_{11,10} \beta, \\
& X_{9,12}=D_{2,7} \alpha^{2}+E_{2,7} \beta^{2}+2 A_{11,6}, \\
& X_{10,10}=A_{9,9} \alpha^{2}+B_{4,7} \beta^{2}+3 D_{2,3}, \\
& X_{10,11}=A_{9,10} \alpha \beta+B_{4,8} \alpha \beta,
\end{aligned}
$$




$$
\begin{aligned}
& X_{10,12}=-A_{9,6} \alpha+3 D_{2,7} \alpha, \\
& X_{11,11}=A_{10,10} \beta^{2}+B_{4,8} \alpha^{2}+3 E_{2,3}, \\
& X_{11,12}=-A_{10,6} \beta+3 E_{2,7} \beta, \\
& X_{12,12}=D_{2,4} \alpha^{2}+E_{4,7} \beta^{2}+3 A_{6,6} \\
& \text { and } \quad X_{i, j}=X_{j, i} \quad(i, j=1,12) .
\end{aligned}
$$

\section{References}

[1] Reissner E, Stavsky Y. Bending and stretching of certain types of heterogeneous aelotropic elastic plates. ASME J Appl Mech 1961;28:402-28

[2] Timoshenko SP, Woinowsky-Krieger S. Theory of plates and shells. New York: Mc-Graw Hill; 1959.

[3] Szilard R. Theory and analysis of plates (classical and numerical methods). New Jersy: Prentice-Hall; 1974.

[4] Reissner E. The effect of transverse shear deformation on the bending of elastic plates. ASME J Appl Mech 1945;12(2): 69-77.

[5] Mindlin RD. Influence of rotary inertia and shear on flexural motions of isotropic, elastic plates. ASME J Appl Mech 1951; $18: 31-8$

[6] Hildebrand FB, Reissner E, Thomas GB. Note on the foundations of the theory of small displacements of orthotropic shells. NACA TN-1833, 1949.

[7] Nelson RB, Lorch DR. A refined theory for laminated orthotropic plates. ASME J Appl Mech 1974;41:177-83.

[8] Librescu L. Elastostatics and kinematics of anisotropic and heterogeneous shell type structures. The Netherlands: Noordhoff; 1975.

[9] Lo KH, Christensen RM, Wu EM. A higher order theory of plate deformation, Part 1: Homogeneous plates. ASME J Appl Mech 1977a;44(4):663-8.

[10] Lo KH, Christensen RM, Wu EM. A higher order theory of plate deformation, Part 2: Laminated plates. ASME J Appl Mech 1977b;44(4):669-76.

[11] Levinson M. An accurate simple theory of the statics and dynamics of elastic plates. Mech Res Commun 1980;7:343.

[12] Murthy MVV. An improved transverse shear deformation theory for laminated anisotropic plates. NASA Technical Paper-1903, 1981.
[13] Whitney JM, Pagano NJ. Shear deformation in heterogeneous anisotropic plates. ASME J Appl Mech 1970;37(4):1031-6.

[14] Kant T. Numerical analysis of thick plates. Comput Meth Appl Mech Eng 1982;31:1-18.

[15] Reddy JN. A simple higher order theory for laminated composite plates. ASME J Appl Mech 1984;51:745-52.

[16] Senthilnathan NR, Lim KH, Lee KH, Chow ST. Buckling of shear deformable plates. AIAA J 1987;25(9):1268-71.

[17] Kant T, Owen DRJ, Zienkiewicz OC. A refined higher order $C^{0}$ plate bending element. Comput Struct 1982;15:177-83.

[18] Pandya BN, Kant T. A consistent refined theory for flexure of a symmetric laminate. Mech Res Commun 1987;14:107-13.

[19] Pandya BN, Kant T. Higher order shear deformable theories for flexure of sandwich plates - finite element evaluations. Int J Solids Struct 1988a;24(12):1267-86.

[20] Pandya BN, Kant T. Flexure analysis of laminated composites using refined higher order $C^{0}$ plate bending elements. Comput Meth Appl Mech Eng 1988b;66:173-98.

[21] Pandya BN, Kant T. A refined higher order generally orthotropic $C^{0}$ plate bending element. Comput Struct 1988c;28:119-33.

[22] Pandya BN, Kant T. Finite element stress analysis of laminated composite plates using higher order displacement model. Compos Sci Technol 1988d;32:137-55.

[23] Kant T, Manjunatha BS. An unsymmetric FRC laminate $C^{0}$ finite element model with 12 degrees of freedom per node. Eng Comput 1988;5(3):300-8.

[24] Kant T, Manjunatha BS. On accurate estimation of transverse stresses in multilayer laminates. Comput Struct 1994;50(3): $351-65$.

[25] Manjunatha BS, Kant T. A comparison of 9 and 16 node quadrilateral elements based on higher order laminate theories for estimation of transverse stresses. J Reinforced Plastics Compos 1992;11(9):968-1002.

[26] Rohwer K. Application of higher order theories to the bending analysis of layered composite plates. Int J Solids Struct 1992; 29(1):105-19.

[27] Noor AK, Burton WS. Assessment of shear deformation theories for multilayered composite plates. Appl Mech Rev 1989;42(1): $1-13$.

[28] Pagano NJ. Exact solutions for rectangular bidirectional composites and sandwich plates. J Compos Mater 1970; 4(1):20-34.

[29] Reddy JN. Energy and variational methods in applied mechanics. New York: Wiley; 1984.

[30] Reddy JN. Mechanics of laminated composite plates, theory and analysis. Boca Raton, FL: CRC Press; 1996. 